Article

\title{
Characterization and Analysis of the Full-Length Transcriptomes of Multiple Organs in Pseudotaxus chienii (W.C.Cheng) W.C.Cheng
}

\author{
Li Liu ${ }^{1}$, Zhen Wang ${ }^{1}$, Yingjuan Su ${ }^{1,2, *}$ and Ting Wang ${ }^{3, *}$ \\ 1 School of Life Sciences, Sun Yat-sen University, Guangzhou 510275, China; liuli11307@163.com (L.L.); \\ zhenwangzhenwang@163.com (Z.W.) \\ 2 Research Institute of Sun Yat-sen University in Shenzhen, Shenzhen 518057, China \\ 3 College of Life Sciences, South China Agricultural University, Guangzhou 510642, China \\ * Correspondence: suyj@mail.sysu.edu.cn (Y.S.); tingwang@scau.edu.cn (T.W.); \\ Tel.: +86-020-84111939 (Y.S.); +86-020-85280185 (T.W.)
}

Received: 12 May 2020; Accepted: 12 June 2020; Published: 17 June 2020

\begin{abstract}
Pseudotaxus chienii, a rare tertiary relict species with economic and ecological value, is a representative of the monotypic genus Pseudotaxus that is endemic to China. P. chienii can adapt well to habitat isolation and ecological heterogeneity under a variety of climate and soil conditions, and is able to survive in harsh environments. However, little is known about the molecular and genetic resources of this long-lived conifer. Herein, we sequenced the transcriptomes of four organs of $P$. chienii using the PacBio Isoform Sequencing and Illumina RNA Sequencing platforms. Based on the PacBio Iso-Seq data, we obtained 44,896, 58,082, 50,485, and 67,638 full-length unigenes from the root, stem, leaf, and strobilus, respectively, with a mean length of $2692 \mathrm{bp}$, and a mean N50 length of $3010.75 \mathrm{bp}$. We then comprehensively annotated these unigenes. The number of organ-specific expressed unigenes ranged from 4393 in leaf to 9124 in strobilus, suggesting their special roles in physiological processes, organ development, and adaptability in the different four organs. A total of 16,562 differentially expressed genes (DEGs) were identified among the four organs and clustered into six subclusters. The gene families related to biotic/abiotic factors, including the TPS, CYP450, and HSP families, were characterized. The expression levels of most DEGs in the phenylpropanoid biosynthesis pathway and plant-pathogen interactions were higher in the root than in the three other organs, suggesting that root constitutes the main organ of defensive compound synthesis and accumulation and has a stronger ability to respond to stress. The sequences were analyzed to predict transcription factors, long non-coding RNAs, and alternative splicing events. The expression levels of most DEGs of $\mathrm{C} 2 \mathrm{H} 2, \mathrm{C} 3 \mathrm{H}, \mathrm{bHLH}$, and bZIP families in the root and stem were higher than those in the leaf and strobilus, indicating that these TFs may play a crucial role in the survival of the root and stem. These results comprise the first comprehensive gene expression profiles obtained for different organs of $P$. chienii. Our findings will facilitate further studies on the functional genomics, adaptive evolution, and phylogeny of $P$. chienii, and lay the foundation for the development of conservation strategies for this endangered conifer.
\end{abstract}

Keywords: Pseudotaxus chienii; PacBio Iso-Seq; transcriptome; multiple organs; phenylpropanoid biosynthesis pathway; plant-pathogen interactions

\section{Introduction}

Pseudotaxus chienii (Taxaceae), a rare tertiary relict species, is a representative of the monotypic genus Pseudotaxus that is endemic to China [1]. The distinguishing feature of this species is the presence of a white aril and two distinct white stomatal bands on the underside of mature leaves [1]. 
This species is a dioecious evergreen woody shrub or tree that grows in subtropical mountain forests [2]. According to the International Union for Conservation of Nature (IUCN) Red List, P. chienii has been classified as a vulnerable species, due to the low conception rate of the female plants, its low seed germination rate, and its slow natural regeneration [3,4]. Populations of P. chienii are sparsely distributed in forest valleys or on cliffs, primarily growing in shallow and acidic soil or in the crevices of rocks [3,5]. P. chienii can adapt to habitat isolation and ecological heterogeneity well, in a variety of climate and soil conditions [5,6]. As an old rare species with a long life, long generation time, and long history, P. chienii is highly valued, and suitable for the investigation of adaptive evolution. Although a previous study on $P$. chienii focused on the use of second-generation sequencing technology to characterize the transcriptome [7], the majority of the studied unigenes were not full-length (FL) cDNA sequences, and did not yield sufficient gene expression profiles of the multiple organs. A few studies have focused on multiple organs in species, showing that organ differentiation is the main driver of transcriptome recombination [8]. In Arabidopsis thaliana, each organ type has a specific expression pattern, and the degree to which organs share expression profiles, is highly correlated with the biological relationship of the organ types [8]. Similar findings have been reported for conifers, such as white spruce (Picea glauca) [9], maritime pine (Pinus pinaster) [10], and Norway spruce (Picea abies) [11]. These findings suggest that the consideration of multiple organs, including the determination of organ-specific expression patterns, is a powerful approach to characterizing the transcriptomic complexity across a whole organism. The current work attempts to fill this gap by studying the multiple organ transcriptomes of P. chienii.

Conifer transcriptome studies are the molecular basis for our understanding of the plant growth, development, and stress adaptation. Conifers are one of the longest living plants on the earth, and have survived through numerous climate oscillations, pest infestations, and natural disasters that have occurred over time [12-14]. They have evolved many sophisticated mechanisms against various environmental stresses, most of which depend on changes in gene expression [15]. Several important candidate genes and metabolic pathways have been identified to respond to biotic and abiotic stresses in some conifer species. Hall et al. [16] used transcriptome assemblies to investigate the defense genes of six tissue/organ types from Pinus banksiana and Pinus contorta, and identified candidate full-length prenyl transferase, terpene synthase (TPS), and cytochrome P450 (CYP450) genes. So far, the majority of the TPSs and CYP450s found in plants have been confirmed to function in plant defense and stress resistance. Conifer trees produce a number of terpenoid metabolites, which act as a chemical barrier against insects and pathogens [17]. CYP450s can promote plant growth and protect plants from stresses through a variety of biosynthetic pathways, such as flavonoids, lignins, phenolics, antioxidants, and phenylpropanoids [18]. Other gene products are important for abiotic stresses (e.g., drought and low-temperature stresses), including dehydrin, endochitinase, heat shock protein (HSP), phenylalanine ammonia-lyase (PAL), and late-embryogenesis abundant (LEA) proteins $[19,20]$. HSPs, as molecular chaperones, can protect plants against stress by reconstructing normal protein conformation [21]. PAL is a key enzyme catalyzing the first step in the phenylpropanoid biosynthesis pathway, which is an important process for the synthesis of defensive compounds [22,23]. Several genes involved in the phenylpropanoid pathway produce physical and chemical barriers against stress, such as the formation of lignin and phenylpropanoid compounds [22]. The plant-pathogen interactions also may play a role in conifer defense, which is an important process that allows plants to avoid infection and achieve immunity [23]. However, despite the importance of these gene families and metabolic pathways in conifer species, their systematic and thorough analysis in P. chienii is still lacking.

Transcriptome analysis could identify the type and number of intracellular key genes and reveal the metabolic pathways at a molecular level [24,25]. At present, several technologies have been applied for transcriptome sequencing. Among these, second-generation sequencing technology (e.g., Illumina RNA Sequencing (Illumina RNA-Seq)) can characterize gene expression levels at a greater sequencing depth [26]. With the advancement of Illumina RNA-Seq technology and low sequencing costs, we have a good opportunity to uncover the transcriptomic sequences of individual species. However, 
the lack of reference genome sequences for some species makes the assembly and annotation of the transcriptome incomplete and error-prone. Furthermore, it is difficult to identify FL transcripts based on the short reads generated by RNA-Seq. With the development of sequencing technology, third-generation sequencing technology (e.g., PacBio Isoform Sequencing (PacBio Iso-Seq)) can now be used to sequence FL or near FL transcripts without assembly, which helps overcome the limitations of second-generation sequencing technology. This technique has the advantages of long read lengths, high accuracy, high sensitivity, and a low degree of bias [27]. To date, PacBio Iso-Seq has been utilized to identify alternative splicing (AS) events, and also a large number of FL transcripts from many species lacking reference genome sequence information [28].

In this study, we combined the PacBio Iso-Seq and Illumina RNA-Seq technologies to reliably perform comprehensive transcriptome analyses and characterize the gene expression profiles in four organs of $P$. chienii. The aims of our study include: (i) generating reference transcriptome sequences for P. chienii from four organ types by using the PacBio Iso-Seq technique; (ii) detecting transcription factors (TFs), long non-coding RNAs (lncRNAs), and AS events; (iii) exploring gene expression patterns and differentially expressed genes (DEGs) among the four organs; (iv) exploring organ-specific unigene expression profiles; and (v) identifying candidate genes and metabolic pathways for adaptation to biotic and abiotic factors. This study will increase our understanding of the FL transcriptome complexity of P. chienii and provide a valuable molecular-level reference for future studies on the functional genomics, adaptive evolution, phylogeny, and conservation of P. chienii and other conifer species.

\section{Results}

\subsection{The Full-Length Sequences of PacBio Iso-Seq}

Based on PacBio Iso-Seq, 3,589,223, 4,242,683, 4,905,002, and 5,427,724 subreads representing $8.21,8.35,11.42$, and $11.22 \mathrm{Gbp}$ were generated for the root, stem, leaf, and strobilus, with a mean length of 2289, 1968, 2328, and 2068 bp, respectively (Table S1). Circular consensus sequences (CCSs) were obtained from the subreads by self-correction (Figure S1). In total, 223,184, 212,703, 325,323, and 331,286 CCSs were obtained for the root, stem, leaf, and strobilus, respectively. By detecting the sequences, 174,922, 175,321, 270,445, and 269,571 were identified as full-length non-chimeric (FLNC) reads for the root, stem, leaf, and strobilus, with a mean length of $2537 \mathrm{bp}$ and a mean N50 length of $2773 \mathrm{bp}$. Based on the iterative clustering for error correction (ICE) algorithm and the polishing of the Arrow algorithm, 80,434, 92,515, 142,159, and 126,538 high-quality (HQ), FL, and polished consensus isoforms were generated for the root, stem, leaf, and strobilus, respectively (Table S1). The polished consensus isoforms were further corrected using Illumina RNA-Seq data (Table S3) to improve the data quality. Finally, after removing the redundant sequences using CD-HIT, 44,896, 58,082, 50,485, and 67,638 unigenes were obtained for the root, stem, leaf, and strobilus, respectively, with a mean length of $2692 \mathrm{bp}$ and mean N50 length of $3010.75 \mathrm{bp}$ (Table S1). Most unigenes had a length ranging from 2000 to $4000 \mathrm{bp}$, accounting for $62.90 \%$ of the total number (Figure 1b). Most of the unigenes were single-isoform genes present in all four organs (Figure 1a). Based on Benchmarking Universal Single-Copy Ortholog (BUSCO) analyses, approximately $76.88 \%$ of the 1440 expected embryophyte genes were identified as complete, suggesting that the integrity of the P. chienii transcriptome was high (Figure S2).

A total of 197,174 reference transcript sequences for P. chienii were generated from four organs using PacBio Iso-Seq, with a $529.06 \mathrm{Mbp}$ total nucleotide bases. The mean length of all transcripts and the mean N50 length were $2683 \mathrm{bp}$ and $3024 \mathrm{bp}$, respectively (Table S2). A total of $288 \mathrm{mRNAs}$ derived from P. chienii were reported in the National Center for Biotechnology Information (NCBI) database. All 197,174 FL transcripts were aligned to 288 mRNAs derived from the NCBI database using BLASTN, and the results revealed 287 (99.65\%) previously reported mRNAs with similarities to our FL transcripts, with sequence identities ranging from $96.89 \%$ to $100 \%$ ( $E$-value $\left.<1.0 \times 10^{-10}\right)$. 
Among them, 267 previously reported mRNAs had a sequence identity of $99 \%$ or more, which suggests that the PacBio FL transcript database we generated for P. chienii has good accuracy.

a

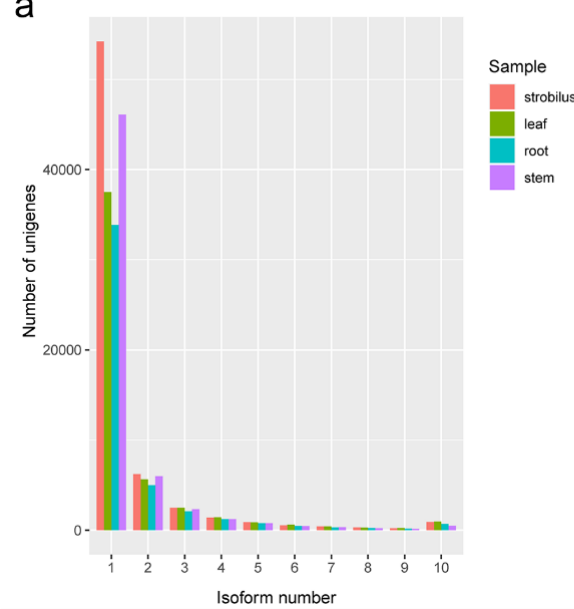

b

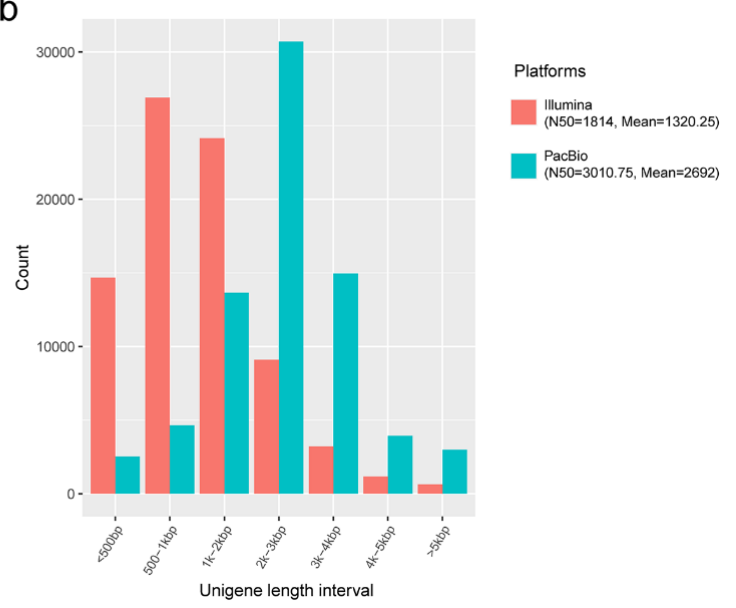

Figure 1. (a) Distribution of isoform numbers for unigenes in four organs of P. chienii; (b) comparison of unigene length distribution using different sequencing platforms.

\subsection{De Novo Assembly of Illumina RNA-Seq Data}

Based on Illumina RNA-Seq, approximately 215 million raw reads were produced from four organs of $P$. chienii. After filtering, for the root, stem, leaf, and strobilus, 51.07, 56.42, 61.11, and 41.45 million clean reads representing 7.66, 8.46, 9.17, and 6.22 Gbp were obtained, with a Q20 of $97.16 \%, 98.03 \%$, $96.9 \%$, and $97.28 \%$, respectively (Table S3). Based on these clean reads, 66,126, 79,842, 52,207, and 60,391 unigenes, respectively, for the root, stem, leaf, and strobilus were assembled de novo, with a mean N50 of $1814 \mathrm{bp}$, and a mean length of $1320.25 \mathrm{bp}$ (Table S4). Most of the unigenes (80.70\%) from Illumina RNA-Seq had a length of less than $2000 \mathrm{bp}$, and only $2332(0.90 \%)$ were more than $5000 \mathrm{bp}$ (Table S5). The comparison of the unigene length distribution for different sequencing platforms is shown in Table S5 and Figure 1b.

\subsection{Functional Annotation}

To derive the most informative and complete information for functional annotation of the unigenes derived from the four organs of $P$. chienii, we performed a similarity search using these sequences in seven public databases, including the NCBI non-redundant protein $(\mathrm{Nr})$, NCBI non-redundant nucleotide (Nt), Swiss-Prot, Pfam protein families, NCBI euKaryotic Ortholog Groups (KOG), Gene Ontology (GO), and Kyoto Encyclopedia of Genes and Genomes (KEGG) databases. For the root, stem, leaf, and strobilus, 40,166 (89.46\%), 49,593 (85.38\%), 47,697 (94.48\%), and 58,654 (86.72\%) unigenes were respectively annotated in at least one of the seven databases, corresponding to 13,610,13,811, 15,489 , and 16,641 unigenes being annotated in all databases (Table 1), indicating that our FL transcripts covered a large number of genes of $P$. chienii, and that most of these were likely functional.

The sequences were compared to those of homologous species by aligning the unigenes using information in the Nr database. The top three homologous species in the root, stem, and leaf were all distributed in Picea sitchensis (9507, 9751, and 11,443 unigenes), Amborella trichopoda (5622, 5296, and 6392 unigenes), and Nelumbo nucifera (3231, 3124, and 3601 unigenes). In the strobilus, the top three homologous species were P. sitchensis (12,019 unigenes), A. trichopoda (5995 unigenes), and Anthurium amnicola (3825 unigenes) (Figure 2). Unsurprisingly, the top homologous species was a conifer. The $E$-value distribution showed that most of the unigenes shared higher homology with their hits from homologous species $\left(E\right.$-value $<1 \times 10^{-100}$ ), and that the distribution patterns of $E$-values were generally similar among the four organs (Figure 2a). 
Table 1. Statistics of annotations of the full-length transcripts from four organs of $P$. chienii with seven databases.

\begin{tabular}{ccccc}
\hline Database & Root & Stem & Leaf & Strobilus \\
\hline Nr & 38,641 & 45,531 & 46,770 & 53,725 \\
Swiss-Prot & 34,034 & 41,986 & 36,644 & 50,386 \\
KEGG & 37,452 & 43,929 & 46,196 & 51,357 \\
KOG & 26,123 & 31,452 & 26,955 & 38,723 \\
GO & 27,425 & 34,770 & 30,132 & 40,467 \\
Nt & 23,665 & 23,851 & 32,373 & 28,089 \\
Pfam & 27,425 & 34,770 & 30,132 & 40,467 \\
At least one database & $40,166(89.46 \%)$ & $49,593(85.38 \%)$ & $47,697(94.48 \%)$ & $58,654(86.72 \%)$ \\
All databases & $13,610(30.31 \%)$ & $13,811(23.78 \%)$ & $15,489(30.68 \%)$ & $16,641(24.60 \%)$ \\
\hline
\end{tabular}

a

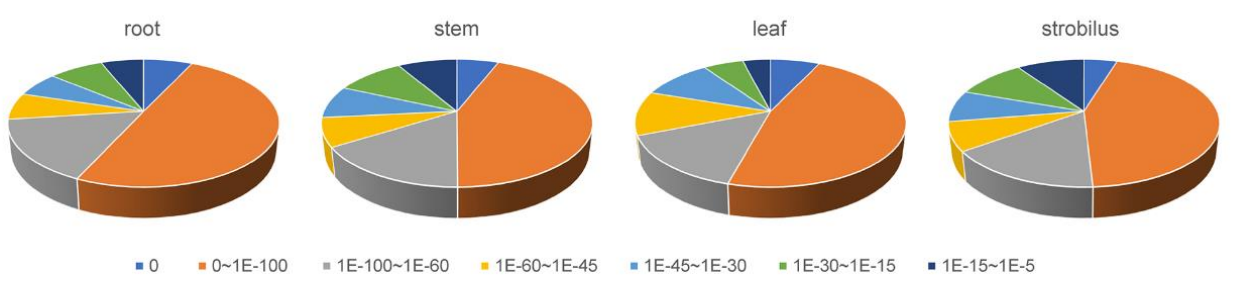

b

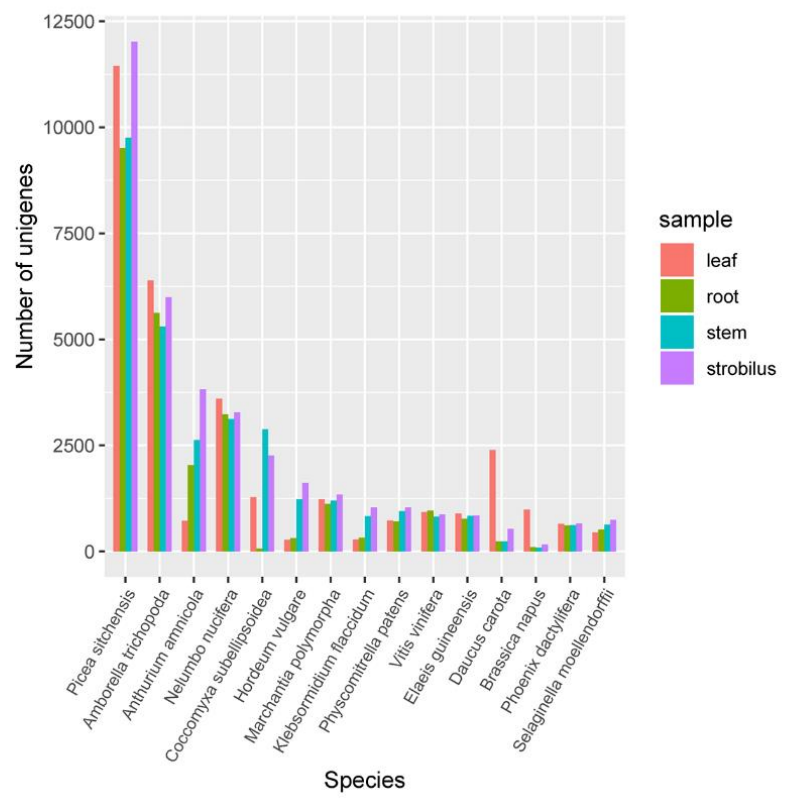

Figure 2. The distribution of homologous species annotated in the NCBI non-redundant protein (Nr) database. (a) The E-value distribution of the hit unigenes; (b) Top 10 hit species for unigenes identified in the four organs of P. chienii.

GO terms were used to functionally classify the $P$. chienii unigenes. For the root, stem, leaf, and strobilus, 27,425 (61.09\%), 34,770 (59.86\%), 30,132 (59.69\%), and 40,467 (59.83\%) unigenes, respectively, were assigned GO terms, which were classified into three main categories, including 17,898, 23,013, 19,977, and 27,076 unigenes from "biological process"; 23,813, 29,877, 25,958, and 34,346 unigenes from "molecular function"; and 8535, 11,567, 9398, and 13,173 unigenes from the "cellular component" (Table S6). For the biological process category, the major subcategories were "metabolic process" (GO: 0008152), "cellular process" (GO: 0009987), and "single-organism process" (GO: 0044699). In the molecular function category, the unigenes involved in "binding" (GO: 0005488), "catalytic activity" (GO: 0003824), and "transporter activity" (GO: 0005215) were highly represented. The major subcategories 
of cellular component were "cell" (GO: 0005623), "cell part" (GO: 0044464), and "membrane" (GO: 0016020) (Figure S3). These representative functions are the most fundamental biological functions necessary for cellular life, and are therefore abundant in all plants. The patterns of the GO annotations for these functions (at level 2) were generally similar across four organs of P. chienii. The category "response to stimulus" (GO: 0050896) is very important for the defense systems of conifer trees, and approximately 8.92\% (2446), 8.53\% (2967), 8.34\% (2512), and 7.79\% (3151) of the unigenes for root, stem, leaf, and strobilus were classified under this category (Table S6). In the GO term "response to stimulus", "cellular response to stimulus" (GO: 0051716), "response to stress" (GO: 0006950), and "response to chemical" (GO: 0042221) were the top 3 annotations in the four organs at GO level 3.

We used the KEGG database to investigate the biological pathways. As with the GO functional classification, the percentages of different KEGG pathway classes were quite similar among the four organs of P. chienii. For the root, stem, leaf, and strobilus, 37,452 (83.42\%), 43,929 (75.63\%), $46,196(91.50 \%)$, and 51,357 (75.93\%) unigenes were assigned to 46 KEGG pathway classes, comprising $367,374,367$, and 375 subclass pathways, respectively (Table S7). "Carbohydrate metabolism" and "signal transduction" were the top two pathways with the greatest number of unigenes in the four organs (Figure S4). For the root, stem, leaf, and strobilus, 1684, 2385, 2397, and 3502 unigenes were assigned to "carbohydrate metabolism", and 2024, 2610, 2021, and 3264 unigenes were assigned to "signal transduction". There was a higher number of unigenes associated with these two pathways in the strobilus than in the other three organs.

For more comprehensive annotation, all unigenes were searched against the KOG database. For the root, stem, leaf, and strobilus, 26,123 (58.19\%), 31,452 (54.15\%), 26,955 (53.39\%), and 38,723 $(57.25 \%)$ unigenes were classified into 26 KOG categories, respectively (Figure 3 ). Similar to the GO classification and KEGG pathways, the distribution patterns of KOG categories were generally similar among the four organs. The undefined functional "general function prediction only", "posttranslational modification, protein turnover, chaperones", and "signal transduction mechanisms" were the top three categories in all four organs. "Defense metabolites" is very important for the physiology and evolution of conifers, and 207, 216, 195, and 267 of the unigenes for the root, stem, leaf, and strobilus were clustered into this category.

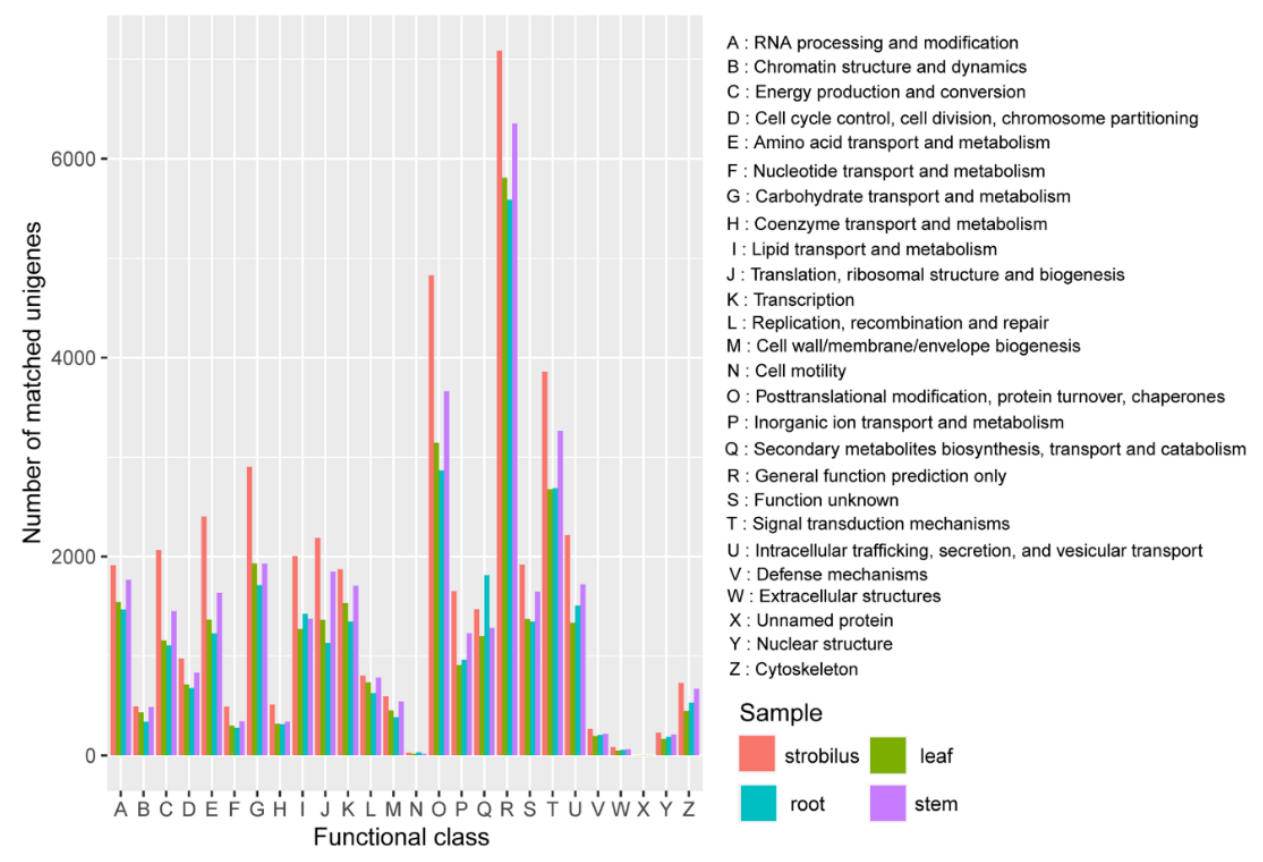

Figure 3. Eukaryotic Ortholog Group (KOG) classification of unigenes. 


\subsection{Identification of CDSs, TFs, and LncRNAs}

For the root, stem, leaf, and strobilus, we identified $45,020,58,539,50,842$, and 68,851 putative coding sequences (CDSs) with a mean length of $1293.5 \mathrm{bp}$, and 26,881,32,398, 29,235, and 39,633 carried complete CDSs. Most $(80.93 \%, 78.80 \%, 78.88 \%$, and $84.49 \%$ for root, stem, leaf, and strobilus) of these CDSs were shorter than $2000 \mathrm{bp}$, and only 2254, 3169, 2972, and $2075(5.01 \%, 5.41 \%, 5.85 \%$, and $3.01 \%)$ were longer than $3000 \mathrm{bp}$. There were $37,204,45,390,42,602$, and 55,482 $5^{\prime}$ untranslated regions (UTRs) and 43,643, 55,685, 49,110, and 66,243 $3^{\prime}$ UTRs for the root, stem, leaf, and strobilus (Table S8).

For the root, stem, leaf, and strobilus, we identified 1678, 2432, 1706, and 2497 TFs from 66 different families, using the iTAK pipeline (Table S9). The top $15 \mathrm{TF}$ families identified in the four organs are shown in Figure 4a. Among them, $\mathrm{C} 2 \mathrm{H} 2, \mathrm{C} 3 \mathrm{H}, \mathrm{bHLH}$, and bZIP were the most commonly represented TF families in all four organs of P. chienii. We found 215, 412, 154, and 381 putative C2H2 TFs in the root, stem, leaf, and strobilus, an obviously highest number than that for other TF families, followed by C3H $(145,181,143$, and 157), bZIP $(85,124,81$, and 154), and bHLH (110, 129, 87, and 121). We further identified the DEGs of the top $15 \mathrm{TF}$ families among the four organs. We found that most DEGs of $\mathrm{C} 2 \mathrm{H} 2, \mathrm{C} 3 \mathrm{H}, \mathrm{bHLH}$, and bZIP families had higher expression levels in the root and stem than those in the leaf and strobilus (Figure $4 \mathrm{~b}$ ). The identification of numerous TFs provides abundant resources for the further research of specific TFs under the various life processes and environmental stresses of P. chienii.

a

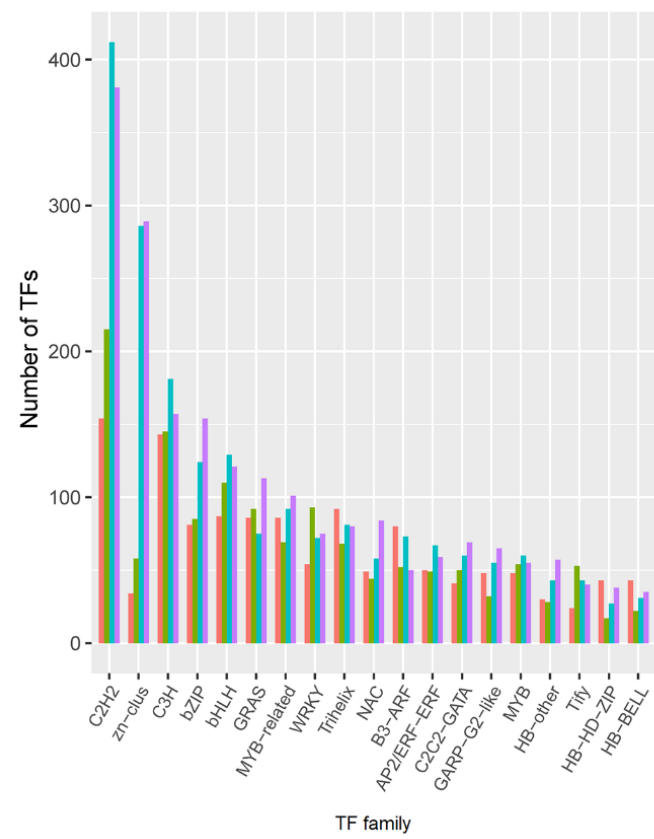

b

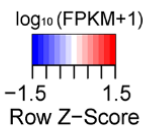

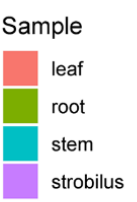

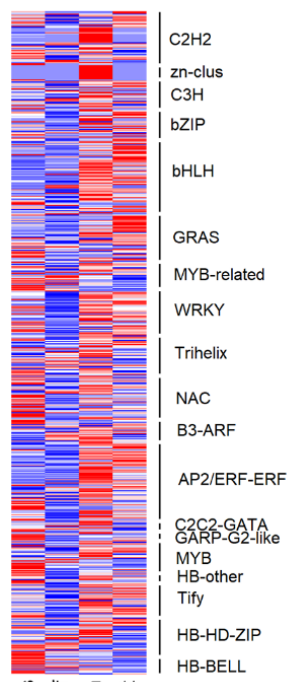

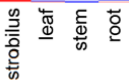

Figure 4. Identification of the transcription factors (TFs). (a) Identification of the top 15 TF families in four organs of P. chienii; (b) a heatmap of differentially expressed genes (DEGs) in the top 15 TF families. A scale indicates the color assigned to $\log _{10}(\mathrm{FPKM}+1)$. Red indicates high expression, and blue indicates low expression.

We used four computational approaches (PLEK, CNCI, CPC, and Pfam) to identify lncRNAs with high confidence. We ultimately identified 2470, 3853, 1218, and 3091 unigenes (accounting for 5.50\%, $6.63 \%, 2.41 \%$, and $4.57 \%$ of the total unigenes, respectively) as putative lncRNAs in the root, stem, leaf, and strobilus, respectively. The number of lncRNAs in leaf was lower than that in the other three organs. The length of the lncRNAs varied from 200 to $9513 \mathrm{bp}$. Most (87.39\%) of these lncRNAs were shorter than $3000 \mathrm{bp}$, and only 101 (0.96\%) were longer than $5000 \mathrm{bp}$ (Figure S5a). The mean length of the lncRNAs was $1382 \mathrm{bp}$, which was shorter than the mean length of all unigenes (2692 bp). The expression 
levels of differentially expressed lncRNAs are shown in a heatmap (Figure S5b). Although we identified many lncRNAs, the functions of these lncRNAs needs to be further characterized.

\subsection{AS Analysis}

For the root, stem, leaf, and strobilus, 10,681, 11,556, 12,485, and 13,206 unique transcript models (UniTransModels) were captured, of which $99.71 \%, 99.81 \%, 99.79 \%$, and $99.73 \%$ had more than one isoform. In total, $11.30 \%$ and $10.73 \%$ of the UniTransModels had more than 10 isoforms in the strobilus and leaf, and these percentages were lower $(7.87 \%$ and $7.75 \%)$ for the stem and root (Figure $5 \mathrm{a}$ ). We identified 461, 475, 559, and 430 AS events in root, stem, leaf, and strobilus covering 3888, 4178, 4035, and 4113 UniTransModels, respectively. Retained introns (RIs) were identified as the predominant AS event in all four organs, and we identified 157, 169, 180, and 152 (accounting for 34.06\%, 35.58\%, $32.20 \%$, and $35.35 \%$ ) RIs in the root, stem, leaf, and strobilus, respectively. The types of alternative $3^{\prime}$ splice sites (A3) $\left(151,158,175\right.$, and 142 for the root, stem, leaf, and strobilus) and alternative $5^{\prime}$ splice sites (A5) $(109,110,162$, and 110 for the root, stem, leaf, and strobilus) were followed. Mutually exclusive exons (MX) and alternative last exons (AL) were the least frequent (Figure 5b).

a

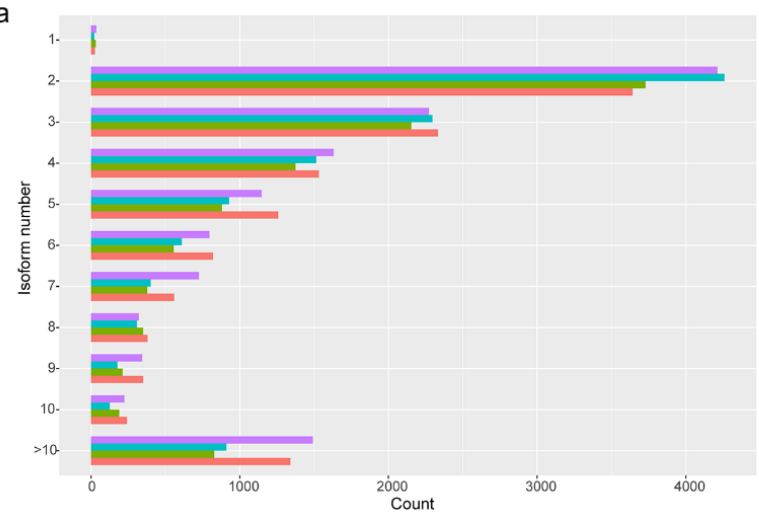

b

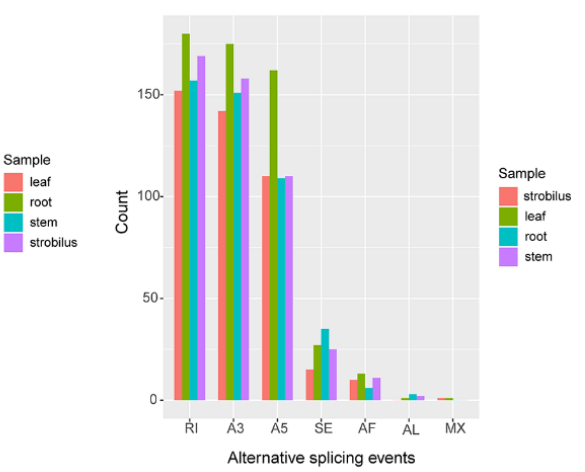

Figure 5. Identification of alternative splicing (AS) events. (a) The distribution of isoform numbers for UniTransModels in four organs; (b) the number of AS events in four organs.

\subsection{Gene Expression Quantification}

The Illumina clean reads of each organ were mapped to the 197,174 FL reference transcript sequences, and the mapping rates were $85.89 \%, 83.97 \%, 89.56 \%$, and $84.82 \%$ for the root, stem, leaf, and strobilus (Table S10), respectively, indicating ideal sequencing and mapping. The expression level of each unigene in each organ was analyzed by estimating the fragments per kilobase of transcript sequence per million base pairs sequenced (FPKM). FPKM interval analysis showed that the FPKM values between 0 and 0.1 accounts for $60 \%$ of all unigenes in four organs, followed by FPKM values between 0.1 and 1, accounting for approximately $16 \%$ of all unigenes; FPKM values were between 1 and 5 in about $11 \%$ of all unigenes; FPKM values were between 5 and 15 in about $4.5 \%$ of all ungenes; FPKM values were between 15 and 60 in about $3 \%$ of all unigenes; and FPKM values were greater than 60 in about $1 \%$ of all unigenes (Figure S6a). Boxplots of the FPKM values in the four organs are shown in Figure S6b. The average FPKMs of the root, stem, leaf, and strobilus were 4.32, 4.79, 5.23, and 5.06, respectively. The unigene expression levels for the root and stem were slightly lower than those for the leaf and strobilus. 


\subsection{DEGs and Functional Enrichment Analysis}

A total of 16,562 DEGs were found among the four organs of P. chienii (Figure 6a). The top 20 enriched pathways of 16,562 DEGs are shown in Figure S7. The 16,562 DEGs clustered into six subclusters through hierarchical cluster analysis (Figure $6 \mathrm{~b}$ ). The expression level of the strobilus organ was used as a control sample. The unigenes in subcluster 1 (3269 unigenes, accounting for $19.74 \%$ ) had the highest expression level in the leaf, compared to those in the other three organs. The KEGG enrichment analysis of the unigenes in subcluster 1 found that most unigenes were involved in "photosynthesis-antenna proteins", "photosynthesis", and "carbon fixation in photosynthetic organisms". In subcluster 2, 3762 unigenes (accounting for 22.71\%) had the lowest expression level in the leaf, and most of these unigenes were enriched in "phenylpropanoid biosynthesis", "flavonoid biosynthesis", and "glutathione metabolism". The unigenes in subcluster 3 (1549 unigenes, accounting for $9.35 \%$ ) had the lowest expression level in the root, while in subcluster 5, 3460 unigenes (accounting for $20.89 \%$ ) had a higher expression level in the root than in the other three organs. The unigenes involved in "flavonoid biosynthesis", "circadian rhythm-plant", and "brassinosteroid biosynthesis" were enriched in subcluster 3 , and most of the unigenes in subcluster 5 were enriched in "diterpenoid biosynthesis", "phenylpropanoid biosynthesis", and "ribosome". The unigenes in subcluster 4 (1614 unigenes, accounting for 9.75\%) had a higher expression level in the strobilus than in the other three organs. The KEGG enrichment analysis of the unigenes in subcluster 4 revealed that most of them had functions in "cyanoamino acid metabolism", "phenylpropanoid biosynthesis", and "ribosome". In subcluster 6, 2908 unigenes (accounting for 17.56\%) had a higher expression level in the stem than in the other three organs. Unigenes in this subcluster functioned mostly in "diterpenoid biosynthesis", "plant hormone signal transduction", and "carotenoid biosynthesis".

To identify the gene expression differences in the different organs (leaf vs. strobilus, leaf vs. root, leaf vs. stem, strobilus vs. root, strobilus vs. stem, and stem vs. root), we compared the paired combinations among the four organs of P. chienii to obtain the upregulated and downregulated unigenes in the latter relative to the former (Table S11). The details of the DEGs for leaf vs. strobilus, leaf vs. root, leaf vs. stem, strobilus vs. root, strobilus vs. stem, and stem vs. root are shown in Table S11. The largest differences were found for leaf vs. root, in which 9660 DEGs were determined, including 4092 upregulated unigenes and 5568 downregulated unigenes, and the number of annotated DEGs was also the greatest (Table S12, Figure 6c). These results indicate that a relatively large number of unigenes of the leaf and root of P. chienii might participate in the growth and development processes. To better explore the main biological functions and metabolic pathways, DEG enrichment analyses were performed in GO terms and KEGG pathways. In leaf vs. strobilus, leaf vs. root, leaf vs. stem, strobilus vs. root, strobilus vs. stem, and stem vs. root, the GO enrichment analysis indicated that 159, 243, 269, 135, 139, and 142 terms, respectively, were significantly enriched. The majority of categories were "catalytic activity" (GO: 0003824) and "metabolic process" (GO: 0008152) (Table S13). These two biological functions are important basic biological functions necessary for cellular life. In the enrichment of the KEGG pathways, 25, 30, 27, 22, 19, and 19 pathways were significantly enriched in the six paired combinations. Large numbers of DEGs were found in "phenylpropanoid biosynthesis" (ko00940) and "starch and sucrose metabolism" (ko00500) (Table S14). It is worth noting that "phenylpropanoid biosynthesis" (ko00940) was enriched in six paired combinations, which plays an important role in the chemical defense of conifer trees. "Phenylpropanoid biosynthesis" was further investigated. These enrichment analysis data provide insights into the metabolite biosynthesis in the four different organs of P. chienii. 
a
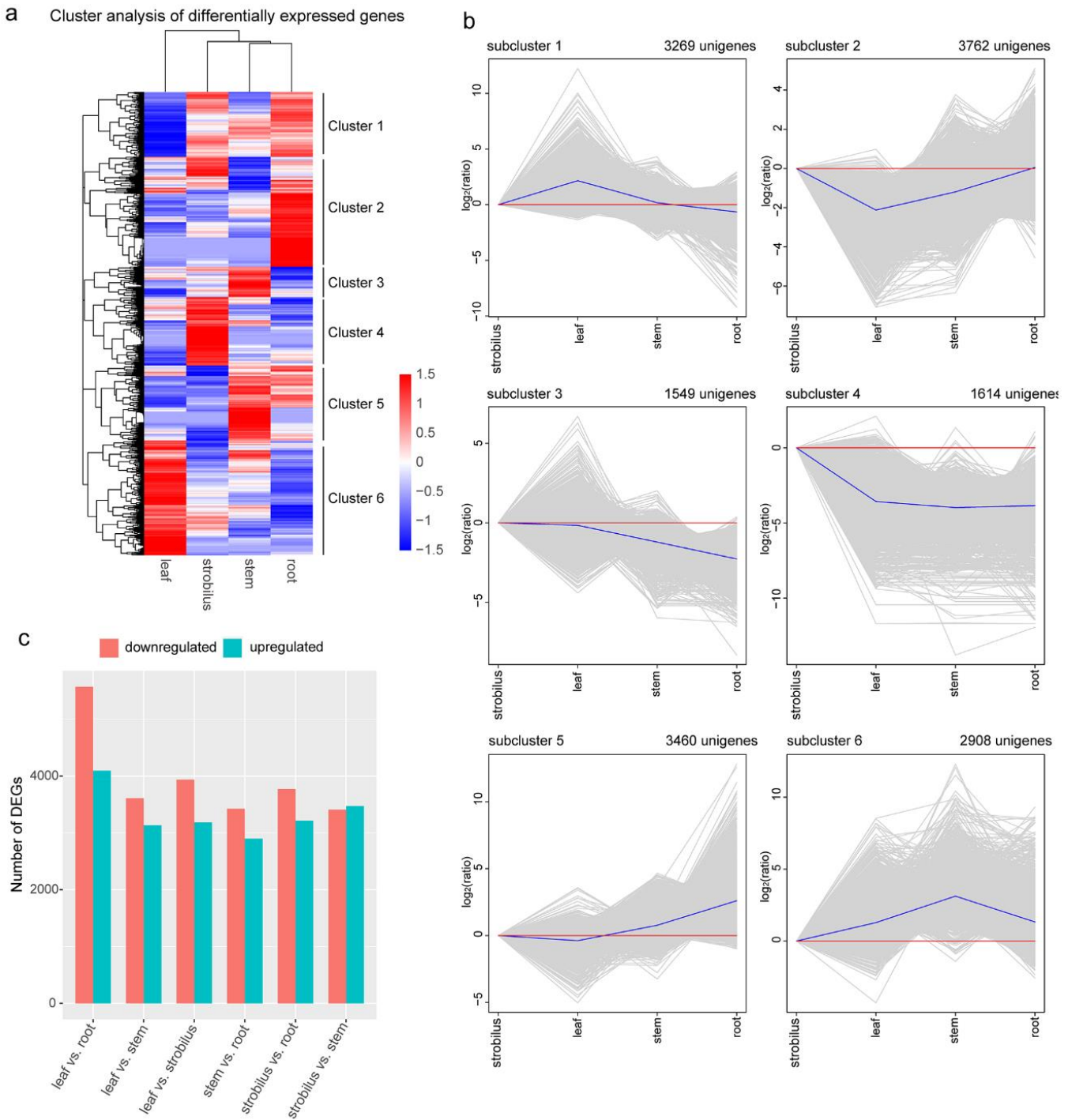

Figure 6. Differentially expressed genes (DEGs) among four organs of P. chienii. (a) A heatmap of the expression patterns of 16,562 DEGs. A scale indicates the color assigned to $\log _{10}(\mathrm{FPKM}+1)$. Red indicates high expression, and blue indicates low expression. (b) The six subclusters of the 16,562 DEGs were clustered. The number of unigenes in each subcluster is shown at the top of the subcluster. The red lines represent the gene expression level of the strobilus. The blue line shows the average values of the relative expression levels in each subcluster, and the gray lines represent the relative expression levels of each unigene. (c) The number of upregulated and downregulated unigenes for leaf vs. strobilus, leaf vs. root, leaf vs. stem, strobilus vs. root, strobilus vs. stem, and stem vs. root.

\subsection{Organ-Specific Expression Unigenes}

We detected 28,901 organ-specific expression unigenes (31.07\%) in the four organs of P. chienii. Of the four organs, the strobilus had the highest proportion of organ-specific unigenes $(9124 ; 9.81 \%)$, followed by the stem $(8140 ; 8.75 \%)$ and $\operatorname{root}(7244 ; 7.79 \%)$, whereas the leaf had the lowest proportion (4393; 4.72\%) (Figure S6c). The number of strobilus-specific unigenes was greater than the other three organs, suggesting that the strobilus has unique characteristics as a reproductive organ. Our discovery of these organ-specific unigenes suggests that they are involved in physiological processes exclusive to certain organs. To explore the main biological functions of these organ-specific unigenes, $\mathrm{GO}$ enrichment analysis was performed on the four organs. In the strobilus, the most enriched GO terms were "transmembrane transport", "single-organism transport", and "single-organism localization". The leaf organ had the least number of organ-specific unigenes, and these unigenes were mainly 
enriched in "single-organism metabolic process", "oxidoreductase activity", and "metabolic process". The functions of these organ-specific unigenes are closely related to the biological characteristics and physiological statuses that are exclusive to certain organs. For instance, in the stem, the most enriched GO terms were "RNA polymerase II transcription factor activity, sequence-specific DNA binding", "transmembrane transport", and "transmembrane transporter activity". Most of these highly enriched GO terms are closely related to the transport function of stem organ. Root-specific unigenes were mainly enriched in "oxidoreductase activity, acting on paired donors, with incorporation or reduction of molecular oxygen", "iron ion binding", and "heme binding". Most of these highly enriched functions are closely related to the function of metal ion binding in root organ.

The KEGG pathway enrichment analysis of organ-specific expression unigenes revealed several significantly enriched pathways in the root, stem, leaf, and strobilus of P. chienii. In the strobilus, the most enriched KEGG pathways were "ribosome", "cyanoamino acid metabolism", and "arachidonic acid metabolism". In the stem, the KEGG pathways of these unigenes were mainly enriched in "citrate cycle (TCA cycle)", "pentose and glucuronate interconversions", and "arginine biosynthesis". As expected, the leaf-specific unigenes were mainly enriched in "carbon fixation in photosynthetic organisms", "photosynthesis-antenna proteins", "glyoxylate and dicarboxylate metabolism", and "photosynthesis". Root-specific unigenes were mainly enriched in "diterpenoid biosynthesis", "ABC transporters", and "phenylpropanoid biosynthesis".

\subsection{Discovery of Gene Families Related to Biotic/Abiotic Factors in P. chienii}

Plants produce a number of TPSs for adaptation to various adverse environments [29]. Based on the annotation information, we identified 237, 67, 94, and 104 putative TPS unigenes for the root, stem, leaf, and strobilus, which included the TPS-d subfamily $(79,53,40$, and 42 putative unigenes for the root, stem, leaf, and strobilus), the TPS-c subfamily (one putative unigene for the strobilus and one for leaf), and unigenes with no subfamily identification (Tables S15 and S16). Those in the TPS- $d$ subfamily were the most numerous and specific to gymnosperms. The majority of TPS sequences in $P$. chienii were homologues of Taiwania cryptomerioides, Chamaecyparis obtusa, and Chamaecyparis formosensis. In P. abies, several TPS genes have been demonstrated to increase insect and pathogen resistance [30]. The alignment of 25 TPS protein-coding sequences from the P. chienii transcriptome showed more than $70 \%$ sequence similarity to the TPSs from P. abies (GenBank accession numbers: AAO73863), and the highest sequence similarity was $84.38 \%$.

We sought to further understand how these TPS sequences relate to a larger family comprised of both angiosperm and gymnosperm TPS sequences. Based on the filtered TPS protein-coding sequences of $P$. chienii and other TPS sequences from gymnosperms and angiosperms, the TPS phylogenetic tree showed that the sequences clearly fell into seven clades (from the TPS-a clade to the TPS-g clade). All TPS sequences from P. chienii were clustered in the TPS-d clade and TPS-e clade, which was consistent with the results of Cupressus gigantea [31]. The TPS-d clade possessed 17 TPS sequences from P. chienii and eight TPS sequences from other gymnosperms. The 17 TPS sequences of P. chienii contained in the TPS- $d$ clade were also identified as belonging to the TPS- $d$ subfamily based on the annotated results. Seven TPS sequences from P. chienii with no subfamily identification and two TPS sequences from Solanum lycopersicum were clustered in the TPS-e clade (Figure 7). 


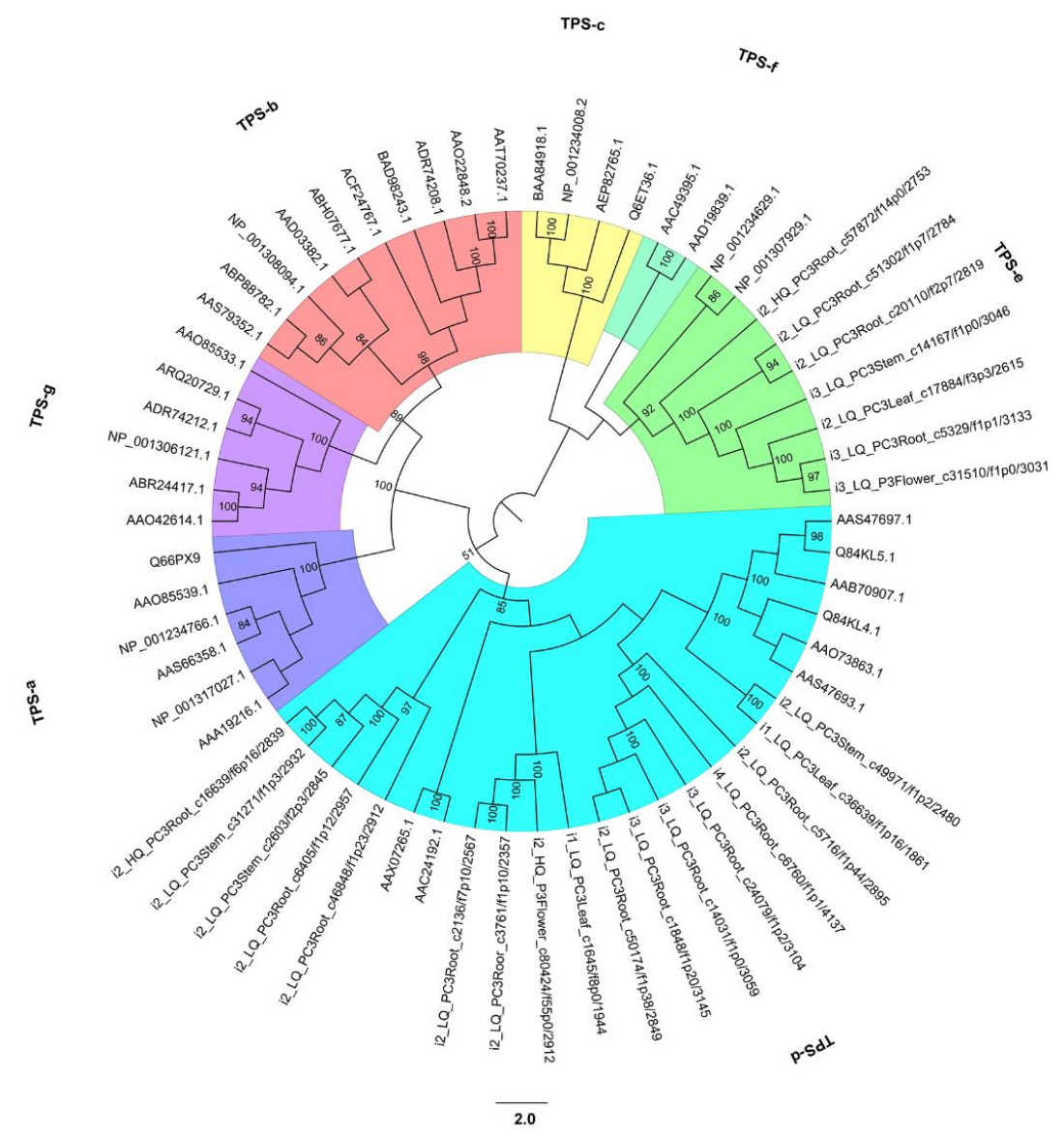

Figure 7. The phylogenetic analysis performed with 62 terpene synthase (TPS) sequences, including the filtered TPS protein-coding sequences of P. chienii (24 sequences) and other TPS sequences from gymnosperms and angiosperms (eight gymnosperm sequences and 30 angiosperm sequences).

The CYP450 is one of the largest gene families in plant species and is important for the metabolism of xenobiotics [18]. CYP450 plays a crucial role in promoting plant development and protecting plants from various environmental stresses [32]. We identified 734, 362, 409, and 487 putative CYP450 unigenes in the root, stem, leaf, and strobilus, respectively (Table S17). The top 10 most abundant subfamilies of CYP450s included CYP725A1, CYP750A1, CYP82C4, CYP716B1, CYP87A3, CYP720B2, CYP716B2, CYP701A6, CYP734A1, and CYP76C2 (Figure S8). Among them, CYP725A1, CYP750A1, CYP716B1, CYP716B2, and CYP720B2 were gymnosperm-specific subfamilies. The most abundant CYP450 subfamily was CYP725A1, with a total of 196 sequences identified throughout the four organs of $P$. chienii. The CYP725A1 subfamily was the most abundant in root organ, with 146 sequences (Figure S8). The second most abundant subfamily was CYP750A1, with a total of 159 sequences identified throughout the four organs of P. chienii, and this subfamily was also the most abundant in root organ, with 66 sequences (Figure S8).

The CYP450 phylogenetic tree with high bootstrap support $>80 \%$ at most nodes showed that 112 putative CYP450 unigenes were classified into nine clans and 30 families and were divided into two categories: type A (71 clan) and non-type A (all other clans) (Figure 8). Within the NJ tree, $47.32 \%$ (53 unigenes) of the 112 putative CYP450 unigenes were type A and belonged to 11 families (CYP719, CYP701, CYP76, CYP75, CYP73, CYP78, CYP703, CYP93, CYP736, CYP92, and CYP82). The remaining $52.68 \%$ (59 unigenes) putative CYP450 unigenes were non-type $\mathrm{A}$ and were distributed among eight clans (727 clan, 97 clan, 86 clan, 72 clan, 710 clan, 74 clan, 51 clan, and 85 clan) and 18 families (CYP727, CYP97, CYP94, CYP86, CYP704, CYP734, CYP735, CYP714, CYP749, CYP710, CYP74, CYP51, CYP720, CYP90, CYP729, CYP716, CYP728, and CYP718), which included a more diverse group of unigenes. Type A CYP450s (71 clan) were identified to be related to the biosynthesis of secondary compounds. The 
non-type A CYP450s were involved in the metabolic pathways of primary products, plant hormones, and secondary products [33]. As a result, a large number of secondary metabolites were synthesized, and functioned as plant growth signals or protected plants from stresses [32].

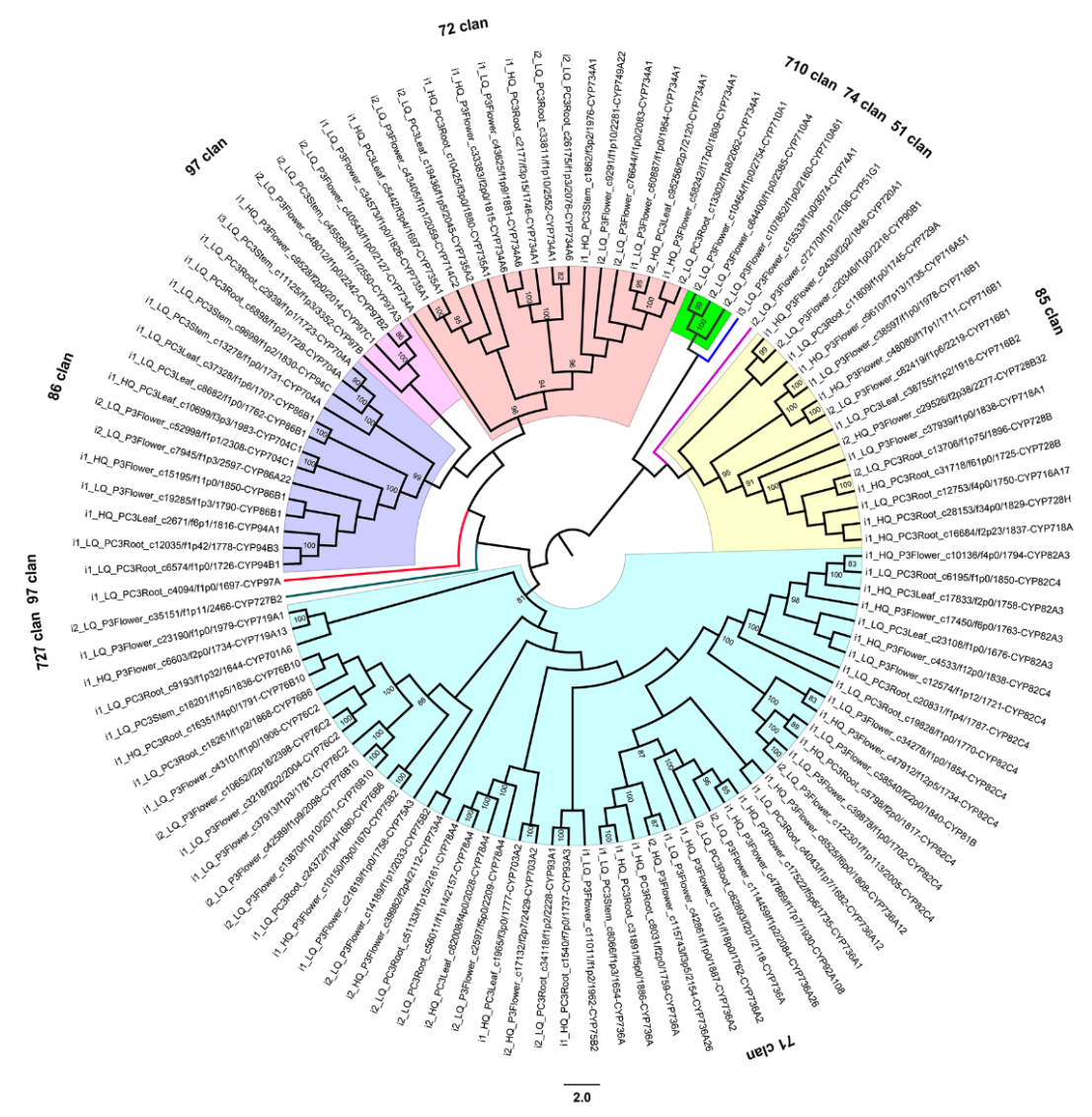

Figure 8. Phylogenetic analyses performed with 112 cytochrome P450 (CYP450) sequences of P. chienii. The classification of CYP450 protein-coding sequences was based on the reference sequences from a CYP450 database established by Nelson (https://drnelson.uthsc.edu/CytochromeP450.html) and the annotation results from the P. chienii transcriptome. Bootstrap support values over $80 \%$ are given.

The resistance and adaptation of plants to adverse environmental conditions are dependent upon the regulation of stress-related genes and gene families. HSPs, as ubiquitous molecular chaperones, are encoded by a multigene family, including five conserved families: small HSP (sHSP)/HSP20, HSP60, HSP70, HSP90, and HSP100 families [34]. In our study, a total of 223, 365, 165, and 464 putative HSP unigenes were identified in the root, stem, leaf, and strobilus, respectively. Among them, HSP70 is the most abundant HSP gene family, with a total of 851 unigenes identified throughout the four organs. The second most abundant family is HSP90, with a total of 248 unigenes identified throughout the four organs. The HSPs showed significant histological specificity in transcription. The identified putative HSP unigenes in leaf were generally less common than those in the other organs; in particular, HSP100 was not detected in leaf organ. The details of the HSP gene families are shown in Tables S15 and S18. In Juglans regia, JrsHSP17.3 can enhance tolerance to abiotic stress, which enhances adaptability in plants [35]. In our study, among the $56 \mathrm{sHSP}$ protein-coding sequences from the $P$. chienii transcriptome, 41 sequences showed a $45.97-85.94 \%$ sequence similarity to JrsHSP17.3 (GenBank accession number: ALR81114.1). The expression patterns of the 56 sHSP unigenes showed that most of sHSP unigenes were expressed in at least one organ, while only six sHSP unigenes were not expressed in any organ. Thirty-one sHSP unigenes $(55.36 \%)$ were predominantly expressed in the stem, and nine sHSP unigenes $(16.07 \%)$ were highly expressed in the leaf, while the expression levels of the 24 sHSP unigenes were lower in the strobilus than in the other three organs (Figure S9). 


\subsection{Characterization of the Unigenes in Phenylpropanoid Biosynthesis Pathway}

Based on the KEGG database, a total of 489 unigenes involved in the phenylpropanoid biosynthesis pathway were identified in four organs of $P$. chienii (Table S19, Figure 9a). For the phenylpropanoid biosynthetic pathway, 11 known enzymes were detected among the four organs, including PAL (phenylalanine ammonia-lyase, 100 unigenes), CYP73A/C4H (trans-cinnamate 4-monooxygenase, 16 unigenes), 4CL (4-coumarate CoA ligase, 51 unigenes), CCR (cinnamoyl-CoA reductase, 18 unigenes), HCT (shikimate $O$-hydroxycinnamoyltransferase, 27 unigenes), CYP98A/C3H (5-O-(4-coumaroyl)-D-quinate 3'-monooxygenase, 22 unigenes), CAD (cinnamyl-alcohol dehydrogenase, 58 unigenes), POD (peroxidase, 120 unigenes), CSE (caffeoyl shikimate esterase, 6 unigenes), COMT (caffeic acid 3-O-methyltransferase, 20 unigenes), and CCoAOMT (caffeoyl-CoA O-methyltransferase, 51 unigenes). Among them, 18 unigenes of PAL, eight of $\mathrm{C} 4 \mathrm{H}$, eight of $4 \mathrm{CL}$, three of $\mathrm{C} 3 \mathrm{H}$, seven of CCR, six of HCT, six of CCOAOMT, four of CSE, nine of COMT, 58 of POD, and 15 of CAD were DEGs (Table S20). The expression patterns of these 142 DEGs associated with the phenylpropanoid biosynthesis pathway in four organs are shown in Figure 9b. Of these 142 DEGs, 82 DEGs were predominantly expressed in the root, including nine of PAL, five of $\mathrm{C} 4 \mathrm{H}$, two of $\mathrm{C} 3 \mathrm{H}$, six of $4 \mathrm{CL}$, two of CCR, six of HCT, four of CSE, six of COMT, four of CCOAOMT, six of CAD, and 32 of POD. However, most of the DEGs (138 DEGs, accounting for 97.18\%) had lower expression levels in the leaf than in the other three organs. In our study, we identified the complete pathway that forms the guaiacyl and $p$-hydroxyphenyl units, which are composed lignins of gymnosperms (Figure 9a).

a

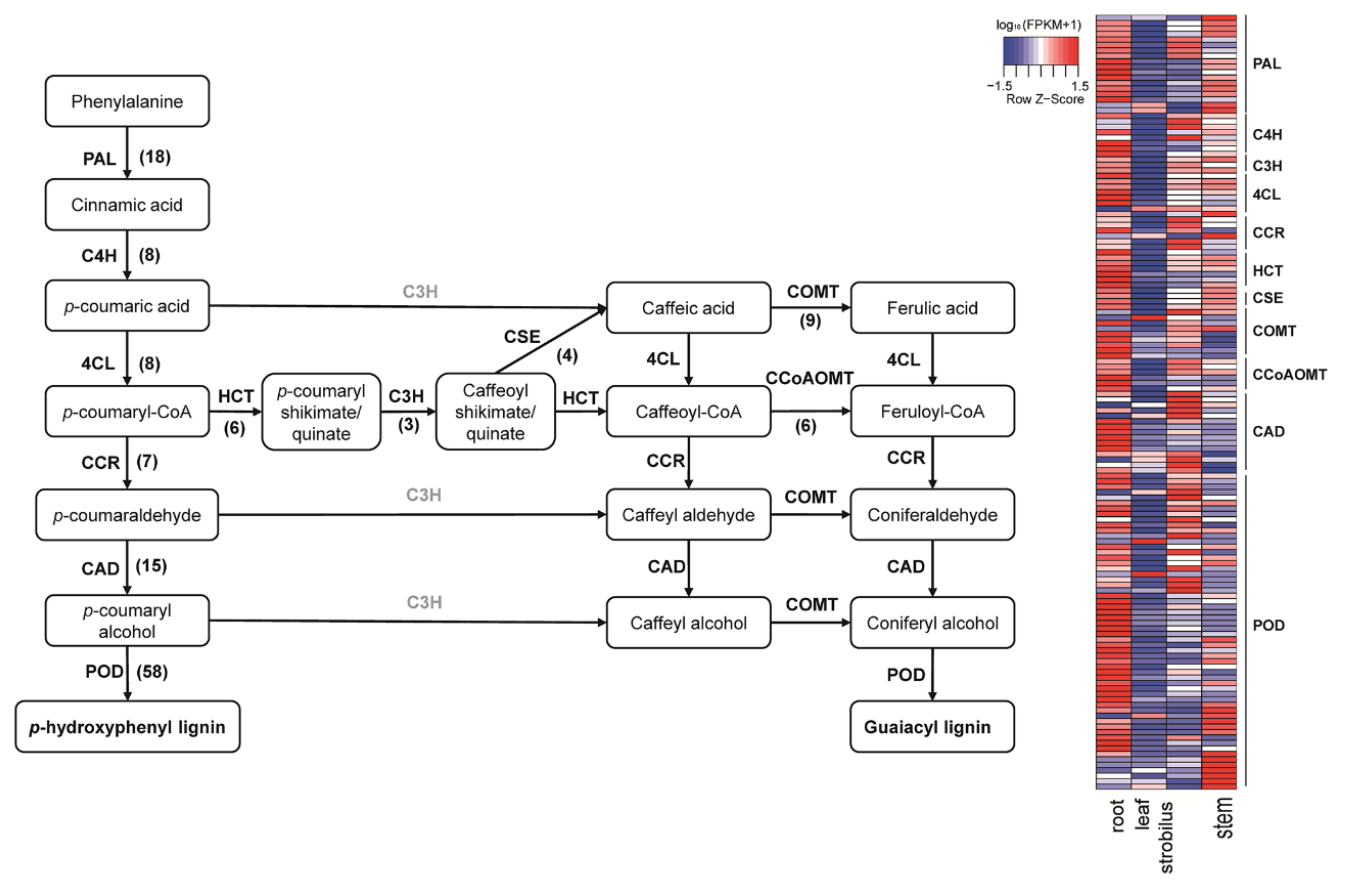

Figure 9. Biosynthesis pathway of phenylpropanoid in P. chienii and the differentially expressed genes (DEGs) involved in phenylpropanoid biosynthesis in four organs of P. chienii. (a) A simplified diagram of the phenylpropanoid biosynthetic pathway. Numbers in brackets represent DEG numbers; (b) a heatmap of the DEGs involved in phenylpropanoid biosynthesis in four organs. PAL: phenylalanine ammonia-lyase; $\mathrm{C} 4 \mathrm{H}$ : trans-cinnamate 4-monooxygenase; 4CL: 4-coumarate CoA ligase; CCR: cinnamoyl-CoA reductase; HCT: shikimate O-hydroxycinnamoyltransferase; C3H: 5-O-(4-coumaroyl)-D-quinate 3'-monooxygenase; CAD: cinnamyl-alcohol dehydrogenase; POD: peroxidase; CSE: caffeoyl shikimate esterase; COMT: caffeic acid 3-O-methyltransferase; CCOAOMT: caffeoyl-CoA O-methyltransferase. 


\subsection{Characterization of the Unigenes in Plant-Pathogen Interactions}

The conifer defense response under stress represents a significant adaptive mechanism [36]. Plants have evolved two main defense systems, pattern-triggered immunity (PTI) and effector-triggered immunity (ETI), which can be activated by plant pathogens [23]. In this study, some PTI- and ETI-related unigenes were detected (Figure 10a).

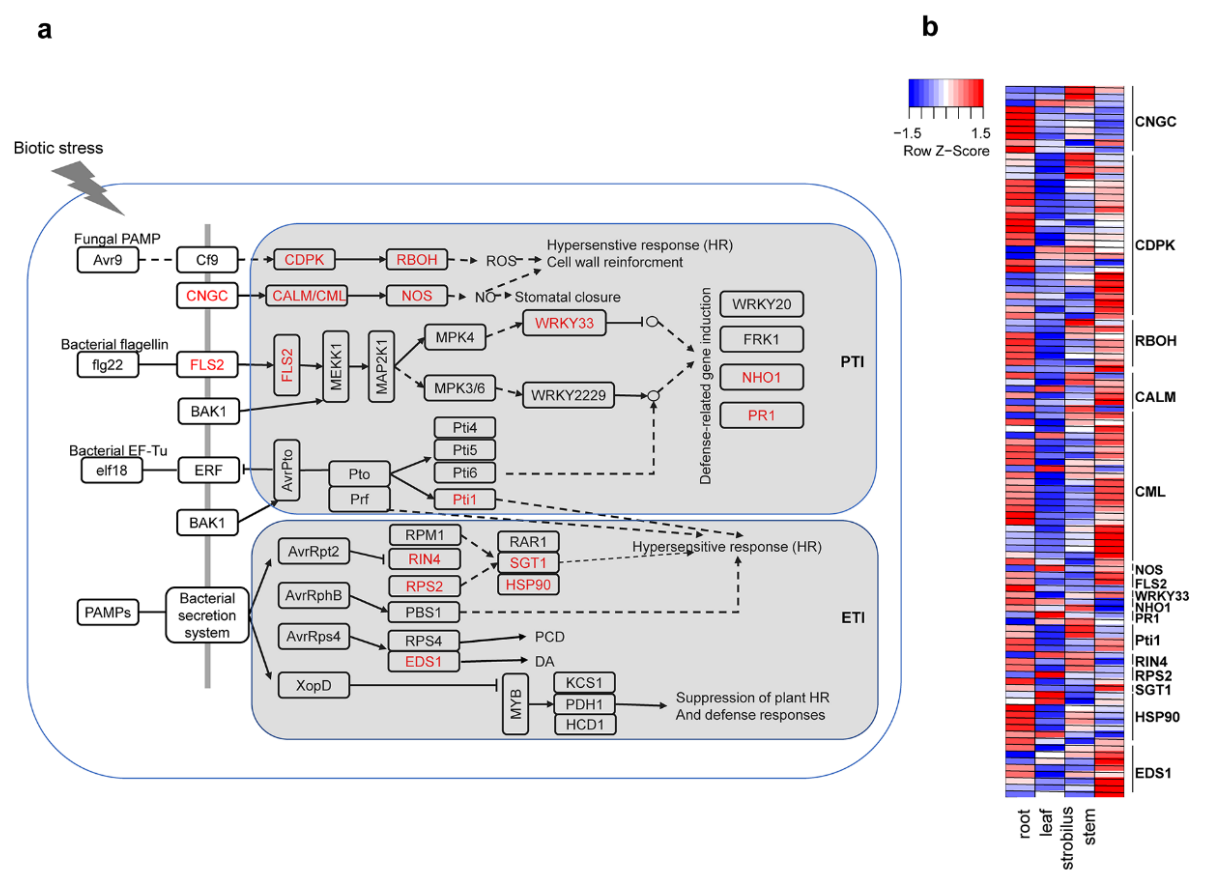

Figure 10. Plant-pathogen interactions in P. chienii and the differentially expressed genes (DEGs) involved in the plant-pathogen interactions in four organs of P. chienii. (a) A simplified diagram of the plant-pathogen interactions; (b) a heatmap of the DEGs involved in the plant-pathogen interactions in four organs. CNGCs: cyclic nucleotide-gated channel; FLS2: LRR receptor-like serine/threonine protein kinase FLS2; BAK1: brassinosteroid insensitive 1-associated receptor kinase; CDPK: calcium-dependent protein kinase; $\mathrm{RBOH}$ : respiratory burst oxidase; CALM: calmodulin; CML: calcium-binding protein CML; NOS: nitric-oxide synthase; PR1: pathogenesis-related protein; NHO1: glycerol kinase; RIN4: RPM1-interacting protein; RPS2: disease resistance protein RPS2; SGT1: suppressor of G2 allele of SKP1; EDS1: enhanced disease susceptibility 1 protein; HSP90: heat shock protein 90.

A total of 827 unigenes involved in the plant-pathogen interactions were detected in the four organs of P. chienii (Table S21). Several genes encoding PRRs were identified, including CNGCs (cyclic nucleotide-gated channel, 127 unigenes), FLS2 (LRR receptor-like serine/threonine protein kinase FLS2, 36 unigenes), and BAK1 (brassinosteroid insensitive 1-associated receptor kinase 1, 1 unigene). Among them, 10 unigenes of CNGCs and three of FLS2 were DEGs. In the PTI system of the plants, CDPK (calcium-dependent protein kinase, 184 unigenes), RBOH (respiratory burst oxidase, 94 unigenes), CALM (calmodulin, 39)/CML (calcium-binding protein CML, 66 unigenes), and NOS (nitric-oxide synthase, seven unigenes) were identified as important processes in the hypersensitive response (HR). FLS2 can activate the WRKY gene, which provides a strong response to pathogen infection and physical wounds in numerous plants [37,38]. In our study, we identified four WRKY33s, one of which was a DEG (Table S22). In addition, PR1 (pathogenesis-related protein 1, six unigenes) and NHO1 (glycerol kinase, 19 unigenes) are important transcriptional regulators of defense-related gene induction. In the ETI system of the plants, RIN4 (RPM1-interacting protein 4, three unigenes), RPS2 (disease resistance protein RPS2, 45 unigenes), SGT1 (suppressor of G2 allele of SKP1, nine unigenes), EDS1 (enhanced disease susceptibility 1 protein, 60 unigenes), and HSP90 (heat shock protein 90, 96 unigenes) were identified. 
Among the 827 unigenes involved in the plant-pathogen interactions, 107 unigenes were DEGs. The expression patterns of these DEGs in the four organs are shown in Figure 10b. Of these 107 DEGs, 46 DEGs were predominantly expressed in the root, including six of CNGC, 12 of CDPK, five of ROBH, one of CALM, nine of CML, one of FLS2, one of NHO1, two of Pti1, one of RIN4, one of RPS2, five of HSP90, and two of EDS. Thirty-six DEGs were predominantly expressed in the stem, including one of CNGC, eight of CDPK, two of ROBH, two of CALM, 13 of CML, two of FLS2, one of WRKY33, one of SGT1, and six of EDS. Together, the expression levels of most DEGs in the plant-pathogen interactions were higher in the root and stem than that in the leaf and strobilus.

\section{Discussion}

\subsection{PacBio Long Reads vs. Illumina Short Reads}

With the development of sequencing technology, PacBio single-molecule real-time (SMRT) sequencing technology provides new insights into FL sequences. This technology is particularly suitable for non-model organisms without reference genome sequences [28]. In our study, using comprehensive analyses with PacBio Iso-Seq and Illumina RNA-Seq transcriptomic data, we provide an overview of the gene expression profiles in four organs of $P$. chienii, by taking advantage of the strong complementarity of these two data types.

A previous study of the P. chienii transcriptome used second-generation transcriptome sequencing technology (Illumina HiSeq2500 sequencing platform) [7]. The author obtained 78,192 unigenes, and the mean length and mean N50 were 622.78 and 1225 bp, respectively. In our study, we obtained 161,585 unigenes and obtained a longer mean length (1130 bp) and mean N50 (1588 bp) using Illumina RNA-Seq. Moreover, we selected four independent organ types, which maximized transcript diversity and provided an overview of the dynamic gene expression profiles in the different organs. The inclusion of more organs in library construction may be conducive to the discovery of rare and organ-specific unigenes. The number of unigenes from Illumina RNA-Seq was larger than the number of PacBio FL unigenes. However, the lengths of unigenes were mainly less than $2000 \mathrm{bp}$ for the former, and longer than $2000 \mathrm{bp}$ for the latter. These results indicate that PacBio Iso-Seq has a better ability to capture long sequences. However, a large number of small size unigenes less than $1000 \mathrm{bp}$ may be missed using PacBio Iso-Seq, due to the technical limitations of size selection in the preparation of cDNA libraries for PacBio Iso-seq [39]. Thus, we used Illumina RNA-Seq data to effectively cover small size unigenes.

\subsection{Functional Annotation}

FL non-redundant sequence information improves the efficiency of functional gene prediction and annotation in plants. In this study, $85.38-94.48 \%$ of the PacBio Iso-Seq unigenes of four independent organ types were successfully annotated by at least one of seven public databases ( $\mathrm{Nt}, \mathrm{Nr}$, Swiss-Prot, Pfam, KOG, GO, and KEGG) (Table 1). These percentages of annotated unigenes based on PacBio Iso-Seq data were higher than for the Illumina RNA-Seq analysis (79.2-81.79\%), indicating that PacBio Iso-Seq data could provide more accurate and effective information for P. chienii transcriptomes. A few unigenes obtained in this study were not annotated in any of the seven public databases. There are several possible reasons: first, there may be a lack of genomic reference information on Taxaceae; second, the unannotated unigenes likely contain non-coding RNA or belong to untranslated regions; third, the unigenes without annotations may be short sequences of absent protein domains. Unannotated unigenes may also be considered novel or species-specific transcript sequences. Our data enriched transcript resources of $P$. chienii offered good performance for discovering novel or uncharacterized transcripts and genes. The GO annotation analyses showed that "metabolic process", "cellular process", "binding", "catalytic activity", "cell", and "cell part" comprise the majority of subcategories, indicating that the organs of $P$. chienii experience active cell metabolism to accumulate sufficient nutrition for the future growth and development of the organs. These GO terms are consistent with the previously investigated Taxus cuspidata transcriptome [25] and the Taxus mairei transcriptome [40]. The KEGG 
annotation analysis showed that "carbohydrate metabolism" and "signal transduction" are the top two pathways with the most abundant unigenes. "Carbohydrate metabolism" can prevent plant cell damage or cell death under low temperatures [41], and "signal transduction" plays an important role in stress response [42].

\subsection{TFs, LncRNAs, and AS Analysis}

FL non-redundant sequences provide much genetic information for transcriptional regulation and posttranscriptional regulation, such as TFs, lncRNAs, and AS events. TFs regulate gene expression via interactions with themselves and other proteins under plant stress response [43]. In our study, we identified 1678, 2432, 1706, and 2497 TFs for the root, stem, leaf, and strobilus, respectively, belonging to 66 different families, which were comparable to those of another conifer species T. cuspidata (1940 TFs), and the model organism A. thaliana (2357 TFs) [25]. C2H2, C3H, bHLH, and bZIP were the most represented TF families in all four organs of $P$. chienii. The phenomenon of the $\mathrm{C} 2 \mathrm{H} 2$ family being the most abundant might be due to a greater number of $\mathrm{C} 2 \mathrm{H} 2$ gene duplication events occurring in the $P$. chienii genome during the evolutionary process, which might have special functions for these genes. $\mathrm{C} 3 \mathrm{H}$ is member of a large family of TFs that modulate the expression of downstream stress-responsive genes in plants [44]. bZIP TFs regulate the processes of stress signaling, flower development, and seed maturation [45]. bHLH TFs are one of the largest regulatory protein families, and are widely distributed in eukaryotes, participating in multiple physiological processes [46]. The expression levels of most DEGs of $\mathrm{C} 2 \mathrm{H} 2, \mathrm{C} 3 \mathrm{H}, \mathrm{bHLH}$, and bZIP families in the root and stem were higher than those in the leaf and strobilus, indicating that these TFs may play a crucial role in the survival of the root and stem. However, MYB-related, NAC, GARP-G2-like, and MYB were more highly expressed in the strobilus than in the other three organs. The expression analysis shows that the TF families exhibit functional differentiation.

LncRNAs, a class of nonprotein-coding unigenes longer than $200 \mathrm{nt}$, are important components of the gene expression regulators functioning in biological processes and plant stress responses [47,48]. The characteristics and genetic patterns of lncRNAs in P. chienii are still unclear. Unlike coding RNAs, lncRNAs lack homology among closely related species. As a result, the information regarding predicted non-coding RNAs for one species is not necessarily useful for other species, which makes prediction and annotation challenging [49]. Recently, increasing numbers of studies have focused on characterizing the functions of lncRNAs in plant species, thereby providing a basis for understanding the functions of IncRNAs in response to environmental change [50]. The more organ types that are used, the more lncRNAs that can be discovered in this species [51]. In our study, IncRNAs were identified in four organ types, with the most in stem and the fewest in leaf. The mean length of lncRNAs (1382 bp) was about double the length of that identified in red clover (Trifolium pratense L.) (665.39 bp) [52]. Additionally, consistent with previous research, the lncRNAs were shorter than the protein-coding mRNAs [50]. Information on these lncRNAs could represent a useful resource for further research on the potential functions and regulatory mechanisms of lncRNAs in P. chienii.

AS is a crucial regulatory mechanism, by which multiple proteins can be produced from a single gene, making it a key factor in greatly increasing the diversity of the transcriptome and proteome of many eukaryotes $[53,54]$. AS represents a posttranscriptional regulatory mechanism that can affect gene expression [55]. During different developmental programs, or under various environmental stresses, the occurrence of AS in plants is high, which has led to an increasing number of studies focusing on revealing the regulatory mechanisms of AS by deep sequencing [56]. Using transcriptome sequencing technologies, AS analysis has been performed in Arabidopsis [56], maize (Zea mays) [57], and soybean (Glycine max) [58]. PacBio long reads contain all of the information originating from a single RNA molecule, giving the data the ability to detect the complexity of an AS event of a species without a reference genome [32]. In our study, we identified 461, 475, 559, and 430 AS events in the root, stem leaf, and strobilus, including RI, A3, A5, SE, AF, AL, and MX AS event types. RI events were found to account for the largest proportion of AS events, which is considered reasonable in terms 
of patterns of AS in plants [56]. However, the number of RIs was estimated to be $34.18 \%$ for all AS events in P. chienii. This proportion is less than that in other published studies on plants ( $40 \%)$ [56]. We suspect that our study underestimates the actual percentage of AS events, since we only used four organ types, corresponding to the same developmental stage under natural growth conditions. We expect that more AS events will be revealed when more organ types are subject to transcriptome analyses during more developmental stages under environmental stress conditions.

\subsection{DEGs and Organ-Specific Unigenes}

A total of 16,562 DEGs were identified among the four organs. It is worth noting that the DEGs and expression profiles we detected come from different organs, not different tissues. KEGG enriched analysis was performed and the top three enriched KEGG pathways were "phenylpropanoid biosynthesis", "flavonoid biosynthesis", and "diterpenoid biosynthesis". The emergence of the phenylpropanoid pathway is one of the important adaptive mechanisms. Phenylpropanoids are precursors to a wide range of compounds, such as isoflavonoids, flavonoids, and stilbenes. These compounds play important roles in plant defense against biotic and abiotic stresses [59]. Flavonoids, a group of powerful antioxidant compounds, were synthesized, starting with the phenylpropanoid pathway. Nakabayashi et al. reported that flavonoids, including flavonols and anthocyanins, have a strong radical scavenging activity, and can protect plants from oxidative and drought stress [60]. During jujube tree (Ziziphus jujuba) infection, the genes involved in phenylpropanoid biosynthesis and flavonoid biosynthesis were significantly upregulated [61]. Diterpenoid compounds are important conifer defense chemicals against herbivores and pathogens [62]. Our results demonstrate that these DEGs play a vital role in enabling adaptation to diverse environments and regulating the growth and development of four different organs in P. chienii.

Organ-specific unigenes were enriched in specific molecular functions and metabolic pathways in different organs, which is consistent with the results published for other plants $[63,64]$. Photosynthesis-related pathways, such as "carbon fixation in photosynthetic organisms", "photosynthesis-antenna proteins", and "photosynthesis", were enriched in leaf organ, which are related to the biological characteristics of leaf organ. Stem-specific unigenes were mainly enriched in the "transmembrane transport" GO term and the "pentose and glucuronate interconversions" pathway. Some studies have suggested that "transmembrane transport" may participate in drought and salt stress responses [42,65], and "pentose and glucuronate interconversions" is involved in the response to anthracnose infection in tea plant (Camellia sinensis) [66]. Root-specific unigenes were significantly enriched in the "ABC transporters" pathway. $A B C$ transporters are involved in a variety of biological processes, enabling plants to adapt to diverse environmental conditions and respond to biotic and abiotic stresses [67]. The absence of $A B C$ transporters affects the expression of many genes related to suberin formation and cuticle metabolism in roots [68]. Strobilus-specific unigenes were significantly enriched in the "arachidonic acid metabolism" and "cyanoamino acid metabolism" pathways. Arachidonic acid is a fungal elicitor that induces a hypersensitive response [69]. "Cyanoamino acid metabolism" might be implicated in $\mathrm{H}_{2} \mathrm{~S}$-dependent drought tolerance in wheat (Triticum aestivum L.) [70]. These significantly enriched molecular functions and pathways of organ-specific unigenes indicate the specific development and environmental response patterns of their corresponding organs. A hierarchical cluster analysis showed the organ-biased expression patterns. The unigenes in different subclusters were involved in the development or the environmental response of the corresponding organs. Together, the expression profiles for genes involved in responses to the environment and growth maintenance were shown to be distinct and organ-specific, and would have significant implications regarding the organ development of P. chienii.

\subsection{Gene Families Related to Biotic/Abiotic Factors in P. chienii}

Terpenoids play a significant role in the chemical and physical defenses in coniferous trees and are produced by the TPS gene family [62]. In our study, we identified 237, 67, 94, and 104 putative 
TPS unigenes in the root, stem, leaf, and strobilus of the P. chienii transcriptome, most of which share high sequence similarity with the TPS sequences from other plants. The identified unigenes of TPS were the most numerous in root organ, suggesting that the TPS gene family may be involved in the defense against root-attacking soil-borne organisms. These numbers are comparable with the numbers of TPSs in angiosperm genomes and other coniferous species (40 in A. thaliana [71], 47 in poplar (Populus trichocarpa) [72], 113 in Eucalyptus [73], 69 in grapevine (Vitis vinifera) [74], 83 in white spruce (P. glauca) [75], 43 in Platycladus orientalis, and 93 in loblolly pine (Pinus taeda) [76]).

Based on the annotation results and phylogenetic analysis, the P. chienii TPS sequences belong to the TPS-c, TPS-d, and TPS-e subfamilies, which is consistent with the previously constructed phylogeny of the gymnosperm TPS family [77]. The majority of gymnosperm TPSs belong to the gymnosperm-specific TPS-d subfamily, and fewer gymnosperm TPSs fall into the subfamilies TPS-c and TPS-e [77]. TPS-c contains copalyl diphosphate synthases (CPS), bifunctional copalyl diphosphate synthases/kaurene synthases (CPS/KS), and other diterpene synthases. TPS-e contains kaurene synthases (KS). The gymnosperm-specific TPS-d subfamily has high functional plasticity, and its great diversity of produced terpenoid compounds may contribute to its defense against stress [78]. The synthesis of the majority of terpene compounds has evolved in plants as a mechanism improved the adaptability of each species to their local ecological niche [77]. The functional characterization of coniferous TPS gene family has been a research hotspot [30,78]. For instance, the expression of many TPS genes involved in volatile emissions and oleoresin defenses can be induced by pathogen infection or herbivore attacks in conifers [79], suggesting that TPS genes have important functions in the defense systems of conifers.

The CYP450 superfamily is one of the largest gene families in plants and mediates reactions involving both primary and secondary metabolites [80]. CYP450 families are involved in various biosynthesis pathways, such as the flavonoid pathway, terpene pathway, salicylic acid pathway, and phenylpropanoid pathway. However, it is difficult to determine the metabolic functions of CYP450s because these enzymes occur in very low quantities and are labile in plants [81]. Therefore, we used transcriptome sequencing to classify CYP450s into unique families and accurately predict their functions. In this study, we identified 734, 362, 409, and 487 putative CYP450 unigenes in the root, stem, leaf, and strobilus respectively. Among the four organs, the unigenes of CYP450 in root were the most abundant. Some of the known gymnosperm-specific CYP450 subfamilies, CYP725A, CYP750A, CYP716B, and CYP720B, were discovered in P. chienii. CYP725A1 is the largest non-type A subfamily, and we identified 146, 16, eight, and 26 putative CYP725A1 unigenes in the root, stem, leaf, and strobilus, respectively. CYP725A1 is a stress-responsive gene, and the molecular regulation of stress-responsive gene expression plays a significant role in the plant response to various stresses [82]. CYP750A1 was found to be the largest type A subfamily, which is consistent with the evidence for T. chinensis [33]. We identified $66,28,27$, and 38 putative CYP750A1 unigenes in the root, stem, leaf, and strobilus, respectively. For the root, stem, leaf, and strobilus, we identified 16, 14, 14, and 19 putative CYP716B1 unigenes and 15, five, 15 , and 12 putative CYP716B2 unigenes in P. chienii. The only confirmed function of the CYP716B gene is the taxoid $9 \alpha$-hydroxylase in Ginkgo biloba [83]. We identified 30, seven, seven, and 11 putative CYP720B2 unigenes in the root, stem, leaf, and strobilus, respectively. In conifers, CYP720B is involved in the formation of diterpene resin acids, which is a major part of the oleoresin defense in conifers [84]. We also identified the CYP73A and CYP98A subfamilies, which are involved in phenylpropanoid biosynthesis and catalyze the first two hydroxylation reactions in the phenolic rings of phenylpropane units. These numbers of CYP450 unigenes in the P. chienii transcriptome are of the same order of magnitude as those in other plant genomes, such as 307 in white spruce (P. glauca) [75], 272 in A. thaliana, 455 in rice (Oryza sativa), and 312 in poplar (P. trichocarpa) [85].

The CYP450 phylogenetic tree analysis provided information about the functional evolution and hinted at some deeper relationships of the CYP450 gene family in P. chienii. For example, 86 clan was adjacent to 97 clan. 85 clan, 51 clan, 74 clan, and 710 clan clustered into a single lineage. These results are consistent with the NJ tree of 142 plant CYP450s constructed by Nelson and Werck-Reichhart [80]. 
Within the CYP450 phylogenetic tree, 112 putative CYP450 unigenes were classified into nine clans, including four multi-family clans (71 clan, 72 clan, 85 clan, and 86 clan) and five single-family clans (51 clan, 74 clan, 97 clan, 710 clan, and 727 clan). The single-family clans restrict themselves from gene duplication, and the enzymes they include are highly conserved with essential functions. These clans are most likely to undergo negative selection [86]. CYP51 is considered to be one of the most conserved and the oldest eukaryotic CYP450s [80]. CYP74 is an atypical CYP450 that is engaged in the formation compounds with signaling or antimicrobial functions [87]. The diversification of CYP450s in multi-family clans parallels the adaptive evolution of land plants. Particularly, the CYP71 clan has expanded rapidly, representing by itself about $50 \%$ of plant CYP450s. Therefore, the CYP71 clan has a great diversity of functions, including being involved in the biosynthesis of lignin, cutin, and sporopollenin [80].

HSPs were originally well-documented in plant responses to high-temperature conditions. However, increasing numbers of investigations have confirmed that HSPs are induced by various abiotic stresses and are involved in plant growth and development [88]. In our study, 56, 23, 851, 248, and 39 unigenes were identified as members of sHSP, HSP60, HSP70, HSP90, and HSP100 families, respectively. HSP70 is the most abundant HSP gene family and is a housekeeping gene functioning in protein folding and protein quality control, followed by HSP90, with a key role in protein degradation and trafficking, cell cycle control, and signal transduction networks $[21,89]$. The features and functions of HSPs have been widely studied in many plants [90]. For example, almost all the CsHSP genes of $C$. sinensis are expressed in one or more organs, and are strongly induced under drought and heat stress [91]. The expression of loblolly pine (P. taeda) HSP genes shows distinctive responses associated with acclimation [92]. In Arabidopsis, the expression of HSP90 is significantly induced under salinity, cold, heat, and heavy metal stress [93,94]. The expression of Chenopodium quinoa Cqhsp70s enhances drought tolerance [95]. Similarly, the functions of sHSP have been widely confirmed. For instance, Salix suchowensis Ssu-sHsps may participate in plant development and stress tolerance by interacting with TFs and functional genes [96]. The expression patterns of the 56 sHSP unigenes of P. chienii indicate that $31 \mathrm{sHSP}$ unigenes $(55.36 \%$ ) are predominantly expressed in the stem, indicating that they may have potential roles in stem development and stress response.

\subsection{Characterization of the Unigenes in Phenylpropanoid Biosynthesis Pathway}

P. chienii is highly adaptable to the diverse environments involved in biotic and abiotic factors $[5,6]$. Metabolites originating from the phenylpropanoid pathway play an important role in conifer defense [97]. We characterized the key genes in the synthesis of the defensive compounds from the phenylpropanoid biosynthesis pathway. These findings are of great significance to the study of the adaptive mechanisms in P. chienii.

Several genes involved in the phenylpropanoid pathway produce physical and chemical barriers against stress, including the formation of lignin and other phenylpropanoids. In the transcriptome sequences of P. chienii, we found FL transcriptome sequences for all key genes in the phenylpropanoid pathway, except for ferulic acid 5-hydroxylase (F5H/CYP84). F5H is necessary to produce syringyl lignin. However, conifers do not produce syringyl lignin [98]. Therefore, $\mathrm{F} 5 \mathrm{H}$ is not expected to be present in P. chienii. In the phenylpropanoid pathway, we identified other CYP450s, including $\mathrm{C} 3 \mathrm{H} / \mathrm{CYP} 98 \mathrm{~A}$ and $\mathrm{C} 4 \mathrm{H} / \mathrm{CYP73A}$. The CYP450 enzyme is the most common derivative involved in the detoxification mechanism of phenylpropanoid biosynthesis. Additionally, of the 142 DEGs, 82 DEGs were predominantly expressed in the root. Therefore, the root constitutes the main organ of phenylpropanoid synthesis and accumulation. Most of the DEGs in phenylpropanoid biosynthesis had relatively low expression levels in the leaf compared to the other three organs, which is consistent with the results for white spruce [99].

The enzymes in the phenylpropanoid pathway of conifers and their involvement in defense mechanisms have been characterized. POD is related to the polymerization of lignin and the oxidation of phenolic compounds. In P. abies, POD conferred resistance against infection by the pathogen Pythium 
dimorphum [100]. In Sitka spruce (P. sitchensis), several known enzymes (including PAL, C3H, C4H, $4 \mathrm{CL}, \mathrm{CCR}, \mathrm{COMT}$, CCoAOMT, and CAD) involved in phenylpropanoid biosynthesis participate in plant defense against insects or wounding [101]. In Larix olgensis Henry, seven DEGs (including 4CL, CCoAOMT, CCR, CAD, PAL, C4H, and POD) related to lignin synthesis were demonstrated to increase disease resistance via cell wall thickening [102]. Lignins from gymnosperms are composed mostly of guaiacyl units, and minor amounts of $p$-hydroxyphenyl units [103]. In our study, we identified the complete pathway that forms guaiacyl and $p$-hydroxyphenyl units (Figure 9). Lignin is essential for the structural integrity of cell walls and the strength and stiffness of the root and stem. Lignin waterproofs the cell walls, enabling water solutes to be transported through the vascular system [97].

\subsection{Characterization of the Unigenes in Plant-Pathogen Interactions}

Conifers are often attacked by pathogens and have developed sophisticated resistance mechanisms to protect themselves. In this study, some PTI- and ETI-related unigenes were detected (Figure 10), indicating that the PTI and ETI defense systems were employed in P. chienii.

Pathogenic signals are transmitted to the cytoplasm by identifying CNGCs, FLS2, and BAK1. The PTI response is then initiated and amplified. In our study, PTI-related unigenes were identified, including CDPK, RBOH, CALM/CML, NOS, FLS2, WRKY33, NHO1, PR1, and Pti1. CALMs are crucial for responses to several biotic and abiotic stresses in plants $[104,105]$. The expression level of tobacco $\mathrm{NtCaM} 13$ was increased in TMV-infected leaves [104]. In this study, 39 unigenes encoding CALMs were identified. Within the cell nucleus, expression of the defense-related gene WRKY 33 and its downstream pathogen-resistance genes NHO1 and PR1 were detected. In general, PTI is enough to defend against most pathogens. However, some pathogens have evolved effector proteins to suppress PTI, which can be overcome by triggering ETI [106]. PTI and ETI share signaling components and outputs, but ETI amplitudes are higher and more persistent, often leading to HR [107]. In our study, several ETI-related unigenes were identified, including RIN4, RPS2, HSP90, SGT1, and EDS1. RPS2 was able to induce disease resistance, and EDS1 could accelerate programmed cell death [108]. The expression levels of most DEGs in the plant-pathogen interactions are higher in the root and stem than that in the leaf and strobilus, suggesting that root and stem organs have a stronger ability to respond to stress.

\section{Materials and Methods}

\subsection{Plant Materials and RNA Isolation}

The root, stem, leaf, and strobilus were collected from P. chienii in 2018 at Bijia Mountain $\left(114^{\circ} 09^{\prime} 42^{\prime \prime}\right.$ E, $26^{\circ} 30^{\prime} 37^{\prime \prime} \mathrm{N}, 1290 \mathrm{~m}$ a.s.l.), Jiangxi Province, China. Four samples of different organs were collected from the same plant. Fresh and healthy samples were washed with purified water and dried with paper towels. All samples were cut into pieces and stored immediately in RNAfixer (BioTeke, Shanghai, China). After collection, all samples were stored at $-20^{\circ} \mathrm{C}$ in a refrigerator until further use.

Total RNA extraction of each sample was performed using a RNAprep Pure Plant Kit, following the protocol of the manufacturer (TianGen, Beijing, China). The purity and concentration of the extracted RNA were determined using a NanoDrop spectrophotometer (Thermo Scientific, Wilmington, DE, USA) and Qubit 2.0 Fluorometer (Invitrogen, Life Technologies, Carlsbad, CA, USA), respectively. RNA integrity was assessed using an Agilent 2100 Bioanalyzer (Agilent Technologies, Santa Clara, CA, USA). High quality RNA with RNA integrity number (RIN) > 8.0 was used for cDNA synthesis and library construction. In total, $1 \mu \mathrm{g}$ RNA of each sample was used for cDNA library construction.

\subsection{PacBio Library Construction and Sequencing}

Total RNA for each of the four organs was constructed library separately according to the PacBio Isoform Sequencing (Iso-Seq) experimental protocol. Poly(A) mRNA was isolated from total RNA using Dynabeads Oligo (dT) magnetic beads (Dynal, Life Technologies, Carlsbad, CA, USA). First-strand cDNA was synthesized from poly(A) mRNA using a Clontech SMARTer PCR cDNA Synthesis Kit 
(Clontech, Mountain View, CA, USA). Then, after optimization of the PCR cycles, large-scale PCR amplification was used to synthesize second-strand cDNA. Size selection was performed with a BluePippin Size Selection System (Sage Science, Beverly, MA, USA) for each sample. Unfiltered fragments and $>4 \mathrm{~kb}$ fragments were equally mixed and used to construct the SMRTbell library with the SMRTBell Template Prep Kit (Pacific Biosciences, Menlo Park, CA, USA). SMRTbell libraries were sequenced on the PacBio Sequel platform (Pacific Biosciences, Menlo Park, CA, USA) using V2 chemistry with $10 \mathrm{~h}$ movies. A total of eight SMRT cells were used for sequencing.

The PacBio Iso-Seq FL transcriptome data were deposited in the Sequence Read Archive (SRA) of NCBI as follows: root: SRR11715805; stem: SRR11715804; leaf: SRR11715803; strobilus: SRR11715802.

\subsection{Illumina Library Construction, Sequencing, and De Novo Assembly}

The Illumina library for each organ was constructed using a NEBNext Ultra ${ }^{\mathrm{TM}}$ RNA Library Prep Kit (NEB, Ipswich, MA, USA), according to the manufacturer's protocol. Sequencing was performed on the Illumina NovaSeq platform, generating paired-end (PE) reads with lengths of $150 \mathrm{bp}$. Illumina RNA-Seq raw reads were processed using in-house Perl scripts. Clean reads were obtained by removing the adapter reads, reads with more than $10 \%$ ambiguous bases ' $\mathrm{N}$ ', and low-quality reads (Qphred $\leq 20$ base with more than 50\%) from the raw reads. Clean reads were de novo assembled with Trinity v.2.4.0 [109] using the following parameters: min_kmer_cov: 2, as well as the remaining default parameters.

The Illumina RNA-Seq transcriptome raw data were deposited in the SRA of NCBI as follows: root: SRR11715801; stem: SRR11715800; leaf: SRR11715799; strobilus: SRR11715798.

\subsection{Preprocessing of the PacBio Iso-Seq Data}

PacBio Iso-Seq raw data were processed with the SMRTlink 5.1 software (http://www.pacb.com/ products-and-services/analytical-sofware/smrt-analysis/). Subreads were obtained from the raw reads using the following parameters: min_length 200, min_readscore 0.65 . CCSs were obtained from the subreads by self-correction using the following parameters: min_length 50, max_drop_fraction 0.8 , no_polish TRUE, min_zscore -9999.0, min_passes 2, min_predicted_accuracy 0.8, max_length 15,000. The CCSs were then classified into FLNC and non-full-length (NFL) reads according to whether or not the $5^{\prime}$-primer, $3^{\prime}$-primer, and poly(A) tail were observed. FLNC reads were performed no isoform-level clustering with the ICE algorithm [110] to obtain FL consensus sequences. The FL consensus sequences were polished by NFL reads using the Arrow algorithm (parameters: hq_quiver_min_accuracy 0.99, bin_by_primer false, bin_size_kb 1, qv_trim_5p 100, qv_trim_3p 30) to obtain HQ, FL, and polished consensus isoforms. Because PacBio reads have a higher frequency of nucleotide errors than Illumina short reads, the polished consensus isoforms were corrected by Illumina RNA-Seq data using the LoRDEC tool [111]. Finally, redundant sequences were removed with CD-HIT (parameters: -c 0.95 -T 6 -G 0 -aL $0.00-a S$ 0.99) to obtain the final transcripts (unigenes) [112]. The final transcripts of multiple organs were merged, and the redundant transcripts were removed based on CD-HIT (parameters: -c 0.95) to obtain the reference transcriptome sequences for P. chienii. The completeness of the transcriptome sequences was evaluated using the BUSCO [113] method with the Embryophyta (ODB9) core gene dataset.

\subsection{Functional Annotation}

The final obtained transcript sequences (unigenes) of the four organs were mapped to seven databases to obtain function annotation information. The NCBI Nr (https:/www.ncbi.nlm.nih.gov/ protein/), KOG (ftp://ftp.ncbi.nih.gov/pub/COG/KOG/), KEGG (http://www.genome.jp/kegg/), and Swiss-Prot (https://www.uniprot.org/uniprot/) databases annotations were performed using DIAMOND v0.8.36 [114] with an $E$-value threshold of $1.0 \times 10^{-5}$. NCBI Nt database annotation was performed using ncbi-blast-2.7.1+ with an $E$-value threshold of $1.0 \times 10^{-5}$. Pfam (https://pfam.xfam.org) database annotation was performed using Hmmscan of the HMMER 3.1 package (http://hmmer.org/) [115]. 
GO (http://www.geneontology.org/) annotations were performed based on the protein annotation information from the Pfam database, using Blast2GO (http://www.blast2go.com) [116] and a script. Based on the KEGG database annotation information, the unigenes involved in the phenylpropanoid biosynthesis pathway and the plant-pathogen interactions were identified in the four organs of P. chienii.

\subsection{Prediction of CDSs, TFs, and LncRNAs}

The ANGEL pipeline was used to predict the CDSs from cDNAs [117]. Firstly, the confident protein sequences of this species and closely related species were used for ANGEL training. Then, the given sequences were predicted using ANGEL. iTAK software was used to identify the plant TFs [118].

Unigenes with lengths greater than $200 \mathrm{nt}$ were selected as IncRNA candidates and further screened via four computational approaches. To obtain a set of high confidence lncRNAs, we calculated the coding potential of each unigene using a series of filtering steps: firstly, PLEK (a predictor of lncRNAs and messenger RNAs based on an improved k-mer scheme) [119] and Coding-Non-Coding Index (CNCI) [120] were employed to categorize the protein-coding and non-coding unigenes and assess coding potential; secondly, the Coding Potential Calculator (CPC) [121] was used to search the sequences via the NCBI eukaryote protein database $\left(E\right.$-value $\left.=1.0 \times 10^{-10}\right)$; finally, we translated each unigene in all three possible frames and identified the known protein family domains documented in the Pfam database according to a Hmmscan homology search with the default parameters of -E 0.001 -domE 0.001. Any unigene with known protein family domains was excluded. As a result, the intersections of predictions of the four filtering tools were considered as the potential lncRNAs of P. chienii.

\subsection{AS Analysis}

We used PacBio Iso-Seq data to reconstruct FL UniTransModels for each of the four organs of P. chienii. Error-corrected non-redundant transcripts were processed with the Coding GENome reconstruction Tool (Cogent v3.1, https:/github.com/Magdoll/Cogent) following the parameters: -dun_use_partial. Firstly, the k-mer profiles of transcripts were created using Cogent by calculating the pairwise distances. Secondly, these transcripts were clustered into families based on their k-mer similarities. Thirdly, the transcript families were further reconstructed into UniTransModels with the de Bruijn graph method. Finally, transcripts were aligned to the UniTransModels using the Genome Mapping and Alignment Program (GMAP-2017-06-20) [122]. AS events were identified using SUPPA (https://github.com/comprna/SUPPA) [123] with default parameters. Different AS event types were identified from the alignments and classified into RI, A3, A5, MX, AL, alternative first exons (AF), and skipping exon (SE).

\subsection{Gene Expression Quantification, DEGs, and Functional Enrichment Analysis}

The clean Illumina reads of each organ sample were mapped to the reference transcriptome sequences using Bowtie 2 [124] of the RSEM (RNA-seq by expectation maximization) software [125] with the end-to-end, sensitive mode and other default parameters, and the readcount value of each unigene for each organ was obtained. Then, all readcounts were normalized to the FPKM [126]. The expression level of each unigene in each sample was determined by estimating the FPKM using RSEM.

The readcount for each unigene in each organ was adjusted with the EdgeR program [127] by one scaling normalized factor. The DEG analysis between each pair combination of different organs (leaf vs. strobilus, leaf vs. root, leaf vs. stem, strobilus vs. root, strobilus vs. stem, and stem vs. root) was performed using the DEGseq of the R package [128] with Poisson's distribution. The $p$ value was adjusted using the $q$ value [129] with the Benjamini-Hochberg approach for controlling the false discovery rate (FDR). Unigenes with $q$ value $<0.005$ and $\mid \log _{2}$ (fold change) $\mid>1$ were considered to be the DEGs. The fold change represents the ratio of the expression level between each two organ samples. Heatmaps were plotted using the gplots 3.0.3 package in R software. Based on the DEGs, 
a hierarchical cluster analysis was performed using the cluster 2.0.5 package in R software. Unigenes expressed only in one organ and not in any other organ were identified as organ-specific unigenes.

GO and KEGG enrichment analyses were performed to identify significantly enriched biological functions and metabolic pathways in DEGs compared against the whole transcriptome background. The GO enrichment analysis of DEGs was implemented via the GOseq R package based on Wallenius non-central hyper geometric distribution [130], in which gene length bias was corrected. GO terms with adjusted $p$ values ( $q$ values) $<0.05$ were considered significantly enriched. The KEGG pathway enrichment analysis of the DEGs was performed using KOBAS (2.0) [131].

\subsection{Identification of the Gene Families Related to Biotic/Abiotic Factors}

Based on functional annotations from publicly available databases $(\mathrm{Nr}$, Swiss-Prot, Pfam, and KOG), the biotic/abiotic factor-related gene families were identified in P. chienii. The number of unigenes annotated for known biotic factor-related gene families (TPS and CYP450) and abiotic factor-related gene families (HSP) were compared among the four organs of P. chienii. The majority of biotic factor-related genes were of the CYP450 gene family. Therefore, the CYP450 and HSP gene families were further investigated. The top 10 most abundant subfamilies of CYP450 in all organs were identified. Some sequences did not feature subfamily identification and were thus not counted. Such genes identified in this study were aligned with the genes conferring resistance in previous studies to assess their homology and similarity in P. chienii with an E-value threshold of $1.0 \times 10^{-10}$.

\subsection{Phylogenetic Analyses}

TPS and CYP450 phylogenetic trees were constructed using the neighbor-joining (NJ) method with the Poisson correction model and 1000 bootstrap replications in MEGA 5.10 [132]. First, the TPS protein-coding sequences were obtained from the P. chienii transcriptome. Second, redundant sequences (95\% similarity) were removed, and only the longest protein-coding sequences were retained. Third, sequences with less than 500 aa and incomplete domains were removed. Fourth, 38 TPS protein sequences of other plants derived from GenBank were obtained, including Abies (2), Pinus (2), Picea (3), Pseudotsuga (1), Arabidopsis (3), Antirrhinum (2), Camellia (1), Clarkia (2), Cucumis (1), Malus (1), Nicotiana (2), Oryza (1), Populus (1), Pyrus (1), Salvia (1), Santalum (1), Solanum (9), and Vitis (4). The TPS protein sequences of these other plants contain all the TPS subfamilies, so they were used to classify the TPS subfamilies of P. chienii. The accession numbers are listed in Table S16. Finally, 24 TPS protein-coding sequences from the $P$. chienii transcriptome and 38 TPS protein sequences of other plants derived from GenBank were used to construct the TPS phylogenetic tree. The filtering of the CYP450 protein-coding sequences is consistent with that of TPS. We ultimately constructed a phylogenetic tree using 112 CYP450 protein-coding sequences. The classification of the CYP450 protein-coding sequences was based on the reference sequences from a CYP450 database established by Nelson (https://drnelson.uthsc.edu/CytochromeP450.html) and the annotation results from the P. chienii transcriptome. The protein-coding sequences for these two gene families were individually aligned with MAFFT (version 7.037) [133] following the default settings. Phylogenetic trees were visualized and annotated using FigTree v1.4.2 [134].

\section{Conclusions}

In summary, we sequenced the full-length transcriptomes in four organs of P. chienii by combining the PacBio Iso-Seq and Illumina RNA-Seq technologies and obtained reference transcriptome sequences for P. chienii. A total of 221,101 unigenes were obtained from four organs and were functionally classified based on a similarity search across seven public databases. Then, CDSs, TFs, IncRNAs, and AS events were detected. The DEGs among the four organs and organ-specific gene expression were profiled. We identified the gene families related to biotic/abiotic factors, including the TPS, CYP450, and HSP families, which possibly participate in the regulation mechanisms underlying ecological adaptability of the four organs of P. chienii. The key genes involved in the phenylpropanoid biosynthesis pathway and 
the plant-pathogen interactions were characterized, and the expression levels of most DEGs in these two pathways were higher in the root than in the other three organs, suggesting that the root constitutes the main organ for the synthesis and accumulation of defensive compound and has a stronger ability to respond to stress and adaptability. These transcriptome data comprise the first comprehensive gene expression profiles across different organs of P. chienii. This study not only provides a practical guide for the transcriptomic analysis of species lacking genomic information but will also facilitate further studies on functional genomics, adaptive evolution, and phylogeny and lay a foundation for the development of conservation strategies for this endangered conifer.

Supplementary Materials: The following are available online at http://www.mdpi.com/1422-0067/21/12/4305/s1, Figure S1. Overview of the data processing pipeline; Figure S2. The results of the transcriptome integrity assessment based on BUSCO using an Embryophyta (ODB9) core gene dataset. The number of Embryophyta gene sets used in this evaluation was 1440; Figure S3. Gene Ontology (GO) classification analysis of unigenes; Figure S4. The Kyoto Encyclopedia of Genes and Genomes (KEGG) pathway classification statistics of the unigenes; Figure S5. The features of long non-coding RNAs (lncRNAs) in four organs. (a) Length distribution of the identified lncRNAs in four organs; (b) A heatmap of identified lncRNAs; Figure S6. Analysis of gene expression in four organs of P. chienii. (a) The FPKM interval distribution in four organs; (b) A boxplot of expression levels in four organs; (c) A venn diagram of the number of unigenes expressed in four organs; Figure S7. The Kyoto Encyclopedia of Genes and Genomes (KEGG) pathway enrichment analysis of 16,562 differentially expressed genes (DEGs); Figure S8. Top 10 most abundant cytochrome P450s (CYP450s) in four organs of P. chienii; Figure S9. A heatmap of the identified heat shock protein (HSP) unigenes. A scale indicates the color assigned to $\log _{10}($ FPKM+1). Red indicates high expression, and blue indicates low expression. Table S1. Summary for the transcriptome data of $P$. chienii using PacBio Iso-Seq; Table S2. Statistics of the reference transcript sequences of $P$. chienii using PacBio Iso-Seq; Table S3. Summary of the reads sequenced using Illumina RNA-Seq; Table S4. Statistics of the unigenes sequenced using Illumina RNA-Seq; Table S5. Comparison of the unigene length distribution for different sequencing platforms; Table S6. Gene Ontology (GO) annotation of the unigenes in four organs of P. chienii; Table S7. Unigene functional classification by Kyoto Encyclopedia of Genes and Genomes (KEGG) in four organs of P. chienii; Table S8. Coding sequences (CDSs) identification for four organs of P. chienii from PacBio Iso-Seq; Table S9. Identification of transcription factors (TFs) in four organs of P. chienii; Table S10. The mapping between the Illumina reads of each sample and the reference transcript sequences generated by PacBio Iso-Seq; Table S11. Differentially expressed genes (DEGs) from the comparisons of the root, stem, leaf, and strobilus; Table S12. The number of upregulated and downregulated differentially expressed genes (DEGs) for leaf vs. strobilus, leaf vs. root, leaf vs. stem, strobilus vs. root, strobilus vs. stem, and stem vs. root; Table S13. Gene Ontology (GO) enrichment analysis of differentially expressed genes (DEGs) from paired combinations of root, stem, leaf, and strobilus; Table S14. Kyoto Encyclopedia of Genes and Genomes (KEGG) pathway enrichment analysis of differentially expressed genes (DEGs) from paired combinations of root, stem, leaf, and strobilus; Table S15. Statistics of the gene families related to biotic/abiotic factors for four organs of P. chienii; Table S16. Statistics of the terpene synthase (TPS) gene family; Table S17. Statistics of the cytochrome P450 (CYP450) gene family; Table S18. Statistics of the heat shock protein (HSP) gene family; Table S19. Statistics of the unigenes in the phenylpropanoid biosynthesis pathway; Table S20. Statistics of the differentially expressed genes (DEGs) in the phenylpropanoid biosynthesis pathway; Table S21. Statistics of the unigenes in the plant-pathogen interactions; Table S22. Statistics of the differentially expressed genes (DEGs) in the plant-pathogen interactions.

Author Contributions: T.W. designed the research and was involved in writing the manuscript; L.L. conducted data analysis and was involved in writing the manuscript; Y.S. helped to supervise the research and was involved in writing the manuscript; Z.W. checked English grammar. All authors read and approved the final version of the manuscript.

Funding: This work was supported by the National Natural Science Foundation of China [31370364, 31570652, 31670200, 31770587, and 31872670], the Natural Science Foundation of Guangdong Province, China [2016A030313320 and 2017A030313122], Science and Technology Planning Project of Guangdong Province, China [2017A030303007], Project of Department of Science and Technology of Shenzhen City, Guangdong, China [JCYJ20160425165447211, JCYJ20170413155402977, JCYJ20170818155249053, and JCYJ20190813172001780], and Science and Technology Planning Project of Guangzhou City, China [201804010389].

Conflicts of Interest: The authors declare no conflict of interest.

\section{References}

1. Fu, L.G.; Li, N.; Mill, R.R. Taxaceae. In Flora of China; Wu, Z.Y., Raven, P.H., Eds.; Science Press: Beijing, China; Missouri Botanical Garden Press: St. Louis, MO, USA, 1999; Volume 4, pp. 89-98.

2. Fu, L.G.; Jin, J.M. Red List of Endangered Plants in China; Science Press: Beijing, China, 1992.

3. Lin, J.X.; He, X.Q.; Hu, Y.S. White berry yew (Pseudotaxus chienii (WC Cheng) WC Cheng). In Conifers: Status Survey and Conservation Action Plan; IUCN: Gland, Switzerland; Cambridge, UK, 1999; p. 106. 
4. Thomas, P.; Yang, Y. Pseudotaxus chienii. The IUCN Red List of Threatened Species 2013: e.T32798A2823334. Available online: https://dx.doi.org/10.2305/IUCN.UK.2013-1.RLTS.T32798A2823334.en (accessed on 16 June 2020).

5. Su, Y.; Wang, T.; Ouyang, P. High genetic differentiation and variation as revealed by ISSR marker in Pseudotaxus chienii (Taxaceae), an old rare conifer endemic to China. Biochem. Syst. Ecol. 2009, 37, 579-588. [CrossRef]

6. Hilfiker, K.; Gugerli, F.; Schutz, J.P.; Rotach, P.; Holderegger, R. Low RAPD variation and female-biased sex ratio indicate genetic drift in small populations of the dioecious conifer Taxus baccata in Switzerland. Conserv. Genet. 2004, 5, 357-365. [CrossRef]

7. Wang, S. Functional Characterization and Evolutionary Analysis of Taxadiene Synthase Gene in Plants. Ph.D. Thesis, Chinese Academy of Forestry, Beijing, China, 2016.

8. Ma, L.; Sun, N.; Liu, X.; Jiao, Y.; Zhao, H.; Deng, X.W. Organ-specific expression of Arabidopsis genome during development. Plant Physiol. 2005, 138, 80-91. [CrossRef] [PubMed]

9. Raherison, E.S.; Giguère, I.; Caron, S.; Lamara, M.; MacKay, J.J. Modular organization of the white spruce (Picea glauca) transcriptome reveals functional organization and evolutionary signatures. New Phytol. 2015, 207, 172-187. [CrossRef]

10. Cañas, R.A.; Li, Z.; Pascual, M.B.; Castro-Rodríguez, V.; Ávila, C.; Sterck, L.; Van de Peer, Y.; Canovas, F.M. The gene expression landscape of pine seedling tissues. Plant J. 2017, 91, 1064-1087. [CrossRef]

11. Yaqoob, N.; Yakovlev, I.A.; Krokene, P.; Kvaalen, H.; Solheim, H.; Fossdal, C.G. Defence-related gene expression in bark and sapwood of Norway spruce in response to Heterobasidion parviporum and methyl jasmonate. Physiol. Mol. Plant Pathol. 2012, 77, 10-16. [CrossRef]

12. Tollefsrud, M.M.; Kissling, R.; Gugerli, F.; Johnsen, O.; Skroppa, T.; Cheddadi, R.; van Der Knaap, W.O.; Latalowa, M.; Terhuerne-Berson, R.; Litt, T.; et al. Genetic consequences of glacial survival and postglacial colonization in Norway spruce: Combined analysis of mitochondrial DNA and fossil pollen. Mol. Ecol. 2008, 17, 4134-4150. [CrossRef]

13. Phillips, M.A.; Croteau, R.B. Resin-based defenses in conifers. Trends Plant Sci. 1999, 4, 184-190. [CrossRef]

14. Kelly, R.; Chipman, M.L.; Higuera, P.E.; Stefanova, I.; Brubaker, L.B.; Hu, F.S. Recent burning of boreal forests exceeds fire regime limits of the past 10,000 years. Proc. Natl. Acad. Sci. USA 2013, 110, 13055-13060. [CrossRef]

15. Rejeb, I.B.; Pastor, V.; Mauch-Mani, B. Plant responses to simultaneous biotic and abiotic stress: Molecular mechanisms. Plants 2014, 3, 458-475. [CrossRef]

16. Hall, D.E.; Yuen, M.M.S.; Jancsik, S.; Quesada, A.L.; Dullat, H.K.; Li, M.; Henderson, H.; Arango-Velez, A.; Liao, N.Y.; Docking, R.T.; et al. Transcriptome resources and functional characterization of monoterpene synthases for two host species of the mountain pine beetle, lodgepole pine (Pinus contorta) and jack pine (Pinus banksiana). BMC Plant Biol. 2013, 13, 80. [CrossRef] [PubMed]

17. Bohlmann, J. Pine terpenoid defences in the mountain pine beetle epidemic and in other conifer pest interactions: Specialized enemies are eating holes into a diverse, dynamic and durable defence system. Tree Physiol. 2012, 32, 943-945. [CrossRef] [PubMed]

18. Schuler, M.A. Plant cytochrome P450 monooxygenases. Crit. Rev. Plant Sci. 1996, 15, 235-284. [CrossRef]

19. Lorenz, W.W.; Sun, F.; Liang, C.; Kolychev, D.; Wang, H.M.; Zhao, X.; Cordonnier-Pratt, M.M.; Pratt, L.H.; Dean, J.F.D. Water stress-responsive genes in loblolly pine (Pinus taeda) roots identified by analyses of expressed sequence tag libraries. Tree Physiol. 2006, 26, 1-16. [CrossRef] [PubMed]

20. Watkinson, J.I.; Sioson, A.A.; Vasquez-Robinet, C.; Shukla, M.; Kumar, D.; Ellis, M.; Heath, L.S.; Ramakrishnan, N.; Chevone, B.; Watson, L.T.; et al. Photosynthetic acclimation is reflected in specific patterns of gene expression in drought-stressed loblolly pine. Plant Physiol. 2003, 133, 1702-1716. [CrossRef]

21. Wang, W.X.; Vinocur, B.; Shoseyov, O.; Altman, A. Role of plant heat-shock proteins and molecular chaperones in the abiotic stress response. Trends Plant Sci. 2004, 9, 244-252. [CrossRef]

22. Dixon, R.A.; Achnine, L.; Kota, P.; Liu, C.J.; Reddy, M.S.S.; Wang, L.J. The phenylpropanoid pathway and plant defence-A genomics perspective. Mol. Plant Pathol. 2002, 3, 371-390. [CrossRef]

23. Hou, S.; Zhang, C.; Yang, Y.; Wu, D. Recent advances in plant immunity: Recognition, signaling, response, and evolution. Biol. Plant. 2013, 57, 11-25. [CrossRef]

24. Zhu, H.; Yu, X.; Xu, T.; Wang, T.; Du, L.; Ren, G.; Dong, K. Transcriptome profiling of cold acclimation in bermudagrass (Cynodon dactylon). Sci. Hortic. 2015, 194, 230-236. [CrossRef] 
25. Kuang, X.; Sun, S.; Wei, J.; Li, Y.; Sun, C. Iso-Seq analysis of the Taxus cuspidata transcriptome reveals the complexity of Taxol biosynthesis. BMC Plant Biol. 2019, 19, 210. [CrossRef]

26. Wang, Z.; Gerstein, M.; Snyder, M. RNA-Seq: A revolutionary tool for transcriptomics. Nat. Rev. Genet. 2009, 10, 57-63. [CrossRef] [PubMed]

27. Roberts, R.J.; Carneiro, M.O.; Schatz, M.C. The advantages of SMRT sequencing. Genome Biol. 2013, $14,405$. [CrossRef] [PubMed]

28. Ning, G.; Cheng, X.; Luo, P.; Liang, F.; Wang, Z.; Yu, G.; Li, X.; Wang, D.; Bao, M. Hybrid sequencing and map finding (HySeMaFi): Optional strategies for extensively deciphering gene splicing and expression in organisms without reference genome. Sci. Rep. 2017, 7, 43793. [CrossRef] [PubMed]

29. Pichersky, E.; Raguso, R.A. Why do plants produce so many terpenoid compounds? New Phytol. 2018, 220, 692-702. [CrossRef]

30. Martin, D.M.; Faldt, J.; Bohlmann, J. Functional characterization of nine Norway spruce TPS genes and evolution of gymnosperm terpene synthases of the TPS-d subfamily. Plant Physiol. 2004, 135, 1908-1927. [CrossRef]

31. Zhou, S.S.; Xing, Z.; Liu, H.; Hu, X.G.; Gao, Q.; Xu, J.; Jiao, S.Q.; Jia, K.H.; Jin, Y.Q.; Zhao, W.; et al. In-depth transcriptome characterization uncovers distinct gene family expansions for Cupressus gigantea important to this long-lived species' adaptability to environmental cues. BMC Genom. 2019, 20, 213. [CrossRef] [PubMed]

32. Xu, Z.; Peters, R.J.; Weirather, J.; Luo, H.; Liao, B.; Zhang, X.; Zhu, Y.; Ji, A.; Zhang, B.; Hu, S.; et al. Full-length transcriptome sequences and splice variants obtained by a combination of sequencing platforms applied to different root tissues of Salvia miltiorrhiza and tanshinone biosynthesis. Plant J. 2015, 82, 951-961. [CrossRef] [PubMed]

33. Liao, W.; Zhao, S.; Zhang, M.; Dong, K.; Chen, Y.; Fu, C.; Yu, L. Transcriptome assembly and systematic identification of novel cytochrome P450s in Taxus chinensis. Front. Plant Sci. 2017, 8, 1468. [CrossRef] [PubMed]

34. Waters, E.R. The evolution, function, structure, and expression of the plant sHSPs. J. Exp. Bot. 2013, 64, 391-403. [CrossRef] [PubMed]

35. Zhai, M.; Sun, Y.; Jia, C.; Peng, S.; Liu, Z.; Yang, G. Over-expression of JrsHSP17. 3 gene from Juglans regia confer the tolerance to abnormal temperature and $\mathrm{NaCl}$ stresses. J. Plant Biol. 2016, 59, 549-558. [CrossRef]

36. Bonello, P.; Gordon, T.R.; Herms, D.A.; Wood, D.L.; Erbilgin, N. Nature and ecological implications of pathogen-induced systemic resistance in conifers: A novel hypothesis. Physiol. Mol. Plant Pathol. 2006, 68, 95-104. [CrossRef]

37. Eulgem, T.; Rushton, P.J.; Robatzek, S.; Somssich, I.E. The WRKY superfamily of plant transcription factors. Trends Plant Sci. 2000, 5, 199-206. [CrossRef]

38. Robatzek, S.; Somssich, I.E. A new member of the Arabidopsis WRKY transcription factor family, AtWRKY6, is associated with both senescence- and defence-related processes. Plant J. 2001, 28, 123-133. [CrossRef]

39. Jo, I.H.; Lee, J.; Hong, C.E.; Lee, D.J.; Bae, W.; Park, S.G.; Ahn, Y.J.; Kim, Y.C.; Kim, J.U.; Lee, J.W.; et al. Isoform sequencing provides a more comprehensive view of the Panax ginseng transcriptome. Genes 2017, 8, 228. [CrossRef]

40. Hao, D.C.; Ge, G.B.; Xiao, P.G.; Zhang, Y.Y.; Yang, L. The first insight into the tissue specific Taxus transcriptome via Illumina second generation sequencing. PLoS ONE 2011, 6, e21220. [CrossRef] [PubMed]

41. Fürtauer, L.; Weiszmann, J.; Weckwerth, W.; Nägele, T. Dynamics of plant metabolism during cold acclimation. Int. J. Mol. Sci. 2019, 20, 5411. [CrossRef] [PubMed]

42. Du, M.; Ding, G.; Cai, Q. The transcriptomic responses of Pinus massoniana to drought stress. Forests 2018, 9, 326. [CrossRef]

43. Wahid, A.; Gelani, S.; Ashraf, M.; Foolad, M.R. Heat tolerance in plants: An overview. Environ. Exp. Bot. 2007, 61, 199-223. [CrossRef]

44. Kodaira, K.S.; Qin, F.; Tran, L.S.P.; Maruyama, K.; Kidokoro, S.; Fujita, Y.; Shinozaki, K.; Yamaguchi-Shinozaki, K. Arabidopsis Cys2/His2 zinc-finger proteins AZF1 and AZF2 negatively regulate abscisic acid-repressive and auxin-inducible genes under abiotic stress conditions. Plant Physiol. 2011, 157, 742-756. [CrossRef]

45. Jakoby, M.; Weisshaar, B.; Dröge-Laser, W.; Vicente-Carbajosa, J.; Tiedemann, J.; Kroj, T.; Parcy, F. bZIP transcription factors in Arabidopsis. Trends Plant Sci. 2002, 7, 106-111. [CrossRef] 
46. Kuijt, S.J.; Greco, R.; Agalou, A.; Shao, J.; CJ’t Hoen, C.; Övernäs, E.; Osnato, M.; Curiale, S.; Meynard, D.; van Gulik, R.; et al. Interaction between the growth-regulating factor and knotted1-like homeobox families of transcription factors. Plant Physiol. 2014, 164, 1952-1966. [CrossRef] [PubMed]

47. Flynn, R.A.; Chang, H.Y. Long noncoding RNAs in cell-fate programming and reprogramming. Cell Stem Cell 2014, 14, 752-761. [CrossRef] [PubMed]

48. Wang, J.; Meng, X.; Dobrovolskaya, O.B.; Orlov, Y.L.; Chen, M. Non-coding RNAs and their roles in stress response in plants. Genom. Proteom. Bioinf. 2017, 15, 301-312. [CrossRef] [PubMed]

49. Yandell, M.; Ence, D. A beginner's guide to eukaryotic genome annotation. Nat. Rev. Genet. 2012, 13, 329-342. [CrossRef]

50. Xu, Q.; Song, Z.; Zhu, C.; Tao, C.; Kang, L.; Liu, W.; He, F.; Yan, J.; Sang, T. Systematic comparison of lncRNAs with protein coding mRNAs in population expression and their response to environmental change. BMC Plant Biol. 2017, 17, 42. [CrossRef]

51. Wang, B.; Regulski, M.; Tseng, E.; Olson, A.; Goodwin, S.; McCombie, W.R.; Ware, D. A comparative transcriptional landscape of maize and sorghum obtained by single-molecule sequencing. Genome Res. 2018, 28, 921-932. [CrossRef]

52. Chao, Y.; Yuan, J.; Li, S.; Jia, S.; Han, L.; Xu, L. Analysis of transcripts and splice isoforms in red clover (Trifolium pratense L.) by single-molecule long-read sequencing. BMC Plant Biol. 2018, 18, 300. [CrossRef]

53. Ben-Dov, C.; Hartmann, B.; Lundgren, J.; Valcarcel, J. Genome-wide analysis of alternative Pre-mRNA splicing. J. Biol. Chem. 2008, 283, 1229-1233. [CrossRef]

54. Barash, Y.; Calarco, J.A.; Gao, W.; Pan, Q.; Wang, X.; Shai, O.; Blencowe, B.J.; Frey, B.J. Deciphering the splicing code. Nature 2010, 465, 53-59. [CrossRef]

55. Reddy, A.S.N. Alternative splicing of pre-messenger RNAs in plants in the genomic era. Annu. Rev. Plant Biol. 2007, 58, 267-294. [CrossRef]

56. Marquez, Y.; Brown, J.W.S.; Simpson, C.; Barta, A.; Kalyna, M. Transcriptome survey reveals increased complexity of the alternative splicing landscape in Arabidopsis. Genome Res. 2012, 22, 1184-1195. [CrossRef] [PubMed]

57. Thatcher, S.R.; Zhou, W.; Leonard, A.; Wang, B.B.; Beatty, M.; Zastrow-Hayes, G.; Zhao, X.; Baumgarten, A.; Li, B. Genome-wide analysis of alternative splicing in Zea mays: Landscape and genetic regulation. Plant Cell 2014, 26, 3472-3487. [CrossRef] [PubMed]

58. Shen, Y.; Zhou, Z.; Wang, Z.; Li, W.; Fang, C.; Wu, M.; Ma, Y.; Liu, T.; Kong, L.A.; Peng, D.L.; et al. Global dissection of alternative splicing in paleopolyploid soybean. Plant Cell 2014, 26, 996-1008. [CrossRef] [PubMed]

59. Ferrer, J.L.; Austin, M.; Stewart, C.J.; Noel, J. Structure and function of enzymes involved in the biosynthesis of phenylpropanoids. Plant Physiol. Biochem. 2008, 46, 356-370. [CrossRef] [PubMed]

60. Nakabayashi, R.; Mori, T.; Saito, K. Alternation of flavonoid accumulation under drought stress in Arabidopsis thaliana. Plant Signal. Behav. 2014, 9, e29518. [CrossRef] [PubMed]

61. Wang, H.; Ye, X.; Li, J.; Tan, B.; Chen, P.; Cheng, J.; Wang, W.; Zheng, X.; Feng, J. Transcriptome profiling analysis revealed co-regulation of multiple pathways in jujube during infection by 'Candidatus Phytoplasma ziziphi'. Gene 2018, 665, 82-95. [CrossRef]

62. Keeling, C.I.; Bohlmann, J. Genes, enzymes and chemicals of terpenoid diversity in the constitutive and induced defence of conifers against insects and pathogens. New Phytol. 2006, 170, 657-675. [CrossRef]

63. Su, W.; Ye, C.; Zhang, Y.; Hao, S.; Li, Q.Q. Identification of putative key genes for coastal environments and cold adaptation in mangrove Kandelia obovata through transcriptome analysis. Sci. Total Environ. 2019, 681, 191-201. [CrossRef]

64. Zhang, Y.; Nyong'A, T.M.; Shi, T.; Yang, P. The complexity of alternative splicing and landscape of tissue-specific expression in lotus (Nelumbo nucifera) unveiled by Illumina-and single-molecule real-time-based RNA-sequencing. DNA Res. 2019, 26, 301-311. [CrossRef]

65. Yang, L.; Jin, Y.; Huang, W.; Sun, Q.; Liu, F.; Huang, X. Full-length transcriptome sequences of ephemeral plant Arabidopsis pumila provides insight into gene expression dynamics during continuous salt stress. BMC Genom. 2018, 19, 717. [CrossRef]

66. Shi, Y.L.; Sheng, Y.Y.; Cai, Z.Y.; Yang, R.; Li, Q.S.; Li, X.M.; Li, D.; Guo, X.Y.; Lu, J.L.; Ye, J.H. Involvement of salicylic acid in anthracnose infection in tea plants revealed by transcriptome profiling. Int. J. Mol. Sci. 2019, 20, 2439. [CrossRef] [PubMed]

67. Sharom, F.J.; Kretzschmar, T.; Burla, B.; Lee, Y.; Martinoia, E.; Nagy, R. Functions of ABC transporters in plants. Essays Biochem. 2011, 50, 145-160. [CrossRef] [PubMed] 
68. Panikashvili, D.; Shi, J.X.; Bocobza, S.; Franke, R.B.; Schreiber, L.; Aharoni, A. The Arabidopsis DSO/ABCG11 transporter affects cutin metabolism in reproductive organs and suberin in roots. Mol. Plant 2010, 3, 563-575. [CrossRef] [PubMed]

69. Bostock, R.; Schaeffer, D.; Hammerschmidt, R. Comparison of elicitor activities of arachidonic acid, fatty acids and glucans from Phytophthora infestans in hypersensitivity expression in potato tuber. Physiol. Mol. Plant Pathol. 1986, 29, 349-360. [CrossRef]

70. Li, H.; Li, M.; Wei, X.; Zhang, X.; Xue, R.; Zhao, Y.; Zhao, H. Transcriptome analysis of drought-responsive genes regulated by hydrogen sulfide in wheat (Triticum aestivum L.) leaves. Mol. Genet. Genom. 2017, 292, 1091-1110. [CrossRef] [PubMed]

71. Aubourg, S.; Lecharny, A.; Bohlmann, J. Genomic analysis of the terpenoid synthase (AtTPS) gene family of Arabidopsis thaliana. Mol. Genet. Genom. 2002, 267, 730-745. [CrossRef]

72. Tuskan, G.A.; DiFazio, S.; Jansson, S.; Bohlmann, J.; Grigoriev, I.; Hellsten, U.; Putnam, N.; Ralph, S.; Rombauts, S.; Salamov, A.; et al. The genome of black cottonwood, Populus trichocarpa (Torr. \& Gray). Science 2006, 313, 1596-1604.

73. Myburg, A.A.; Grattapaglia, D.; Tuskan, G.A.; Hellsten, U.; Hayes, R.D.; Grimwood, J.; Jenkins, J.; Lindquist, E.; Tice, H.; Bauer, D.; et al. The genome of Eucalyptus grandis. Nature 2014, 510, 356-362. [CrossRef]

74. Martin, D.M.; Aubourg, S.; Schouwey, M.B.; Daviet, L.; Schalk, M.; Toub, O.; Lund, S.T.; Bohlmann, J. Functional annotation, genome organization and phylogeny of the grapevine (Vitis vinifera) terpene synthase gene family based on genome assembly, FLcDNA cloning, and enzyme assays. BMC Plant Biol. 2010, 10, 226. [CrossRef]

75. Warren, R.L.; Keeling, C.I.; Yuen, M.M.S.; Raymond, A.; Taylor, G.A.; Vandervalk, B.P.; Mohamadi, H.; Paulino, D.; Chiu, R.; Jackman, S.D.; et al. Improved white spruce (Picea glauca) genome assemblies and annotation of large gene families of conifer terpenoid and phenolic defense metabolism. Plant J. 2015, 83, 189-212. [CrossRef]

76. Hu, X.G.; Liu, H.; Jin, Y.; Sun, Y.Q.; Li, Y.; Zhao, W.; El-Kassaby, Y.A.; Wang, X.R.; Mao, J.F. De novo transcriptome assembly and characterization for the widespread and stress-tolerant conifer Platycladus orientalis. PLoS ONE 2016, 11, e0148985. [CrossRef] [PubMed]

77. Chen, F.; Tholl, D.; Bohlmann, J.; Pichersky, E. The family of terpene synthases in plants: A mid-size family of genes for specialized metabolism that is highly diversified throughout the kingdom. Plant J. 2011, 66, 212-229. [CrossRef] [PubMed]

78. Keeling, C.I.; Weisshaar, S.; Ralph, S.G.; Jancsik, S.; Hamberger, B.; Dullat, H.K.; Bohlmann, J. Transcriptome mining, functional characterization, and phylogeny of a large terpene synthase gene family in spruce (Picea spp.). BMC Plant Biol. 2011, 11, 43. [CrossRef] [PubMed]

79. Miller, B.; Madilao, L.L.; Ralph, S.; Bohlmann, J. Insect-induced conifer defense. White pine weevil and methyl jasmonate induce traumatic resinosis, de novo formed volatile emissions, and accumulation of terpenoid synthase and putative octadecanoid pathway transcripts in Sitka spruce. Plant Physiol. 2005, 137, 369-382. [CrossRef]

80. Nelson, D.; Werck-Reichhart, D. A P450-centric view of plant evolution. Plant J. 2011, 66, 194-211. [CrossRef]

81. Kim, J.A.; Roy, N.S.; Lee, I.H.; Choi, A.Y.; Choi, B.S.; Yu, Y.S.; Park, N.I.; Park, K.C.; Kim, S.; Yang, H.S.; et al. Genome-wide transcriptome profiling of the medicinal plant Zanthoxylum planispinum using a single-molecule direct RNA sequencing approach. Genomics 2019, 111, 973-979. [CrossRef]

82. Kai, G.; Jiang, J.; Zhao, D.; Zhao, L.; Zhang, L.; Li, Z.; Guo, B.; Sun, X.; Miao, Z.; Tang, K. Isolation and expression profile analysis of a new cDNA encoding 5-alpha-taxadienol-10-beta-hydroxylase from Taxus media. J. Plant Biochem. Biotechnol. 2006, 15, 1-5. [CrossRef]

83. Zhang, N.; Han, Z.; Sun, G.; Hoffman, A.; Wilson, I.W.; Yang, Y.; Gao, Q.; Wu, J.; Xie, D.; Dai, J.; et al. Molecular cloning and characterization of a cytochrome P450 taxoid 9a-hydroxylase in Ginkgo biloba cells. Biochem. Biophys. Res. Commun. 2014, 443, 938-943. [CrossRef]

84. Ro, D.K.; Arimura, G.I.; Lau, S.Y.; Piers, E.; Bohlmann, J. Loblolly pine abietadienol/abietadienal oxidase PtAO (CYP720B1) is a multifunctional, multisubstrate cytochrome P450 monooxygenase. Proc. Natl. Acad. Sci. USA 2005, 102, 8060-8065. [CrossRef]

85. Nelson, D.R. Plant cytochrome P450s from moss to poplar. Phytochem. Rev. 2006, 5, 193-204. [CrossRef]

86. Vasav, A.; Barvkar, V. Phylogenomic analysis of cytochrome P450 multigene family and their differential expression analysis in Solanum lycopersicum L. suggested tissue specific promoters. BMC Genom. 2019, $20,116$. [CrossRef] [PubMed] 
87. Hughes, R.K.; De Domenico, S.; Santino, A. Plant cytochrome CYP74 family: Biochemical features, endocellular localisation, activation mechanism in plant defence and improvements for industrial applications. ChemBioChem 2009, 10, 1122-1133. [CrossRef] [PubMed]

88. Park, C.J.; Seo, Y.S. Heat shock proteins: A review of the molecular chaperones for plant immunity. Plant Pathol. J. 2015, 31, 323-333. [CrossRef] [PubMed]

89. Mayer, M.; Bukau, B. Hsp70 chaperones: Cellular functions and molecular mechanism. Cell. Mol. Life Sci. 2005, 62, 670. [CrossRef]

90. Lopes-Caitar, V.S.; de Carvalho, M.C.; Darben, L.M.; Kuwahara, M.K.; Nepomuceno, A.L.; Dias, W.P.; Abdelnoor, R.V.; Marcelino-Guimarães, F.C. Genome-wide analysis of the Hsp20 gene family in soybean: Comprehensive sequence, genomic organization and expression profile analysis under abiotic and biotic stresses. BMC Genom. 2013, 14, 577. [CrossRef]

91. Chen, J.; Gao, T.; Wan, S.; Zhang, Y.; Yang, J.; Yu, Y.; Wang, W. Genome-wide identification, classification and expression analysis of the HSP gene superfamily in tea plant (Camellia sinensis). Int. J. Mol. Sci. 2018, 19, 2633. [CrossRef]

92. Heath, L.S.; Ramakrishnan, N.; Sederoff, R.R.; Whetten, R.W.; Chevone, B.I.; Struble, C.A.; Jouenne, V.Y.; Chen, D.W.; van Zyl, L.; Grene, R. Studying the functional genomics of stress responses in loblolly pine with the Expresso microarray experiment management system. Comp. Funct. Genom. 2002, 3, 226-243. [CrossRef]

93. Milioni, D.; Hatzopoulos, P. Genomic organization of $h s p 90$ gene family in Arabidopsis. Plant Mol. Biol. 1997, 35, 955-961. [CrossRef]

94. Krishna, P.; Gloor, G. The Hsp90 family of proteins in Arabidopsis thaliana. Cell Stress Chaperones 2001, 6, 238-246. [CrossRef]

95. Liu, J.; Wang, R.; Liu, W.; Zhang, H.; Guo, Y.; Wen, R. Genome-wide characterization of heat-shock protein 70s from Chenopodium quinoa and expression analyses of Cqhsp70s in response to drought stress. Genes 2018, 9, 35. [CrossRef]

96. Li, J.; Zhang, J.; Jia, H.; Yue, Z.; Lu, M.; Xin, X.; Hu, J. Genome-wide characterization of the sHsp gene family in Salix suchowensis reveals its functions under different abiotic stresses. Int. J. Mol. Sci. 2018, 19, 3246. [CrossRef] [PubMed]

97. Boerjan, W.; Ralph, J.; Baucher, M. Lignin biosynthesis. Annu. Rev. Plant Biol. 2003, 54, 519-546. [CrossRef]

98. Neale, D.B.; Wegrzyn, J.L.; Stevens, K.A.; Zimin, A.V.; Puiu, D.; Crepeau, M.W.; Cardeno, C.; Koriabine, M.; Holtz-Morris, A.E.; Liechty, J.D.; et al. Decoding the massive genome of loblolly pine using haploid DNA and novel assembly strategies. Genome Biol. 2014, 15, R59. [CrossRef] [PubMed]

99. Lamara, M.; Parent, G.J.; Giguère, I.; Beaulieu, J.; Bousquet, J.; MacKay, J.J. Association genetics of acetophenone defence against spruce budworm in mature white spruce. BMC Plant Biol. 2018, 18, 231. [CrossRef] [PubMed]

100. Fossdal, C.G.; Sharma, P.; Lonneborg, A. Isolation of the first putative peroxidase cDNA from a conifer and the local and systemic accumulation of related proteins upon pathogen infection. Plant Mol. Biol. 2001, 47, 423-435. [CrossRef]

101. Ralph, S.G.; Yueh, H.; Friedmann, M.; Aeschliman, D.; Zeznik, J.A.; Nelson, C.C.; Butterfield, Y.S.N.; Kirkpatrick, R.; Liu, J.; Jones, S.J.M.; et al. Conifer defence against insects: Microarray gene expression profiling of Sitka spruce (Picea sitchensis) induced by mechanical wounding or feeding by spruce budworms (Choristoneura occidentalis) or white pine weevils (Pissodes strobi) reveals large-scale changes of the host transcriptome. Plant Cell Environ. 2006, 29, 1545-1570.

102. Hu, X.; Yang, J.; Li, C. Transcriptomic response to nitric oxide treatment in Larix olgensis Henry. Int. J. Mol. Sci. 2015, 16, 28582-28597. [CrossRef]

103. Huang, H.H.; Xu, L.L.; Tong, Z.K.; Lin, E.P.; Liu, Q.P.; Cheng, L.J.; Zhu, M.Y. De novo characterization of the Chinese fir (Cunninghamia lanceolata) transcriptome and analysis of candidate genes involved in cellulose and lignin biosynthesis. BMC Genom. 2012, 13, 648. [CrossRef]

104. Yamakawa, H.; Mitsuhara, I.; Ito, N.; Seo, S.; Kamada, H.; Ohashi, Y. Transcriptionally and post-transcriptionally regulated response of 13 calmodulin genes to tobacco mosaic virus-induced cell death and wounding in tobacco plant. Eur. J. Biochem. 2001, 268, 3916-3929. [CrossRef]

105. Yuenyong, W.; Chinpongpanich, A.; Comai, L.; Chadchawan, S.; Buaboocha, T. Downstream components of the calmodulin signaling pathway in the rice salt stress response revealed by transcriptome profiling and target identification. BMC Plant Biol. 2018, 18, 335. [CrossRef] 
106. Khan, M.; Subramaniam, R.; Desveaux, D. Of guards, decoys, baits and traps: Pathogen perception in plants by type III effector sensors. Curr. Opin. Microbiol. 2016, 29, 49-55. [CrossRef]

107. Chiang, Y.H.; Coaker, G. Effector triggered immunity: NLR immune perception and downstream defense responses. Arab. Book 2015, 2015, e0183. [CrossRef]

108. Axtell, M.J.; Staskawicz, B.J. Initiation of RPS2-specified disease resistance in Arabidopsis is coupled to the AvrRpt2-directed elimination of RIN4. Cell 2003, 112, 369-377. [CrossRef]

109. Grabherr, M.G.; Haas, B.J.; Yassour, M.; Levin, J.Z.; Thompson, D.A.; Amit, I.; Adiconis, X.; Fan, L.; Raychowdhury, R.; Zeng, Q. Trinity: Reconstructing a full-length transcriptome without a genome from RNA-Seq data. Nat. Biotechnol. 2011, 29, 644. [CrossRef] [PubMed]

110. Gordon, S.P.; Tseng, E.; Salamov, A.; Zhang, J.; Meng, X.; Zhao, Z.; Kang, D.; Underwood, J.; Grigoriev, I.V.; Figueroa, M.; et al. Widespread polycistronic transcripts in fungi revealed by single-molecule mRNA sequencing. PLoS ONE 2015, 10, e0132628. [CrossRef] [PubMed]

111. Salmela, L.; Rivals, E. LoRDEC: Accurate and efficient long read error correction. Bioinformatics 2014, 30, 3506-3514. [CrossRef] [PubMed]

112. Fu, L.; Niu, B.; Zhu, Z.; Wu, S.; Li, W. CD-HIT: Accelerated for clustering the next-generation sequencing data. Bioinformatics 2012, 28, 3150-3152. [CrossRef]

113. Simão, F.A.; Waterhouse, R.M.; Ioannidis, P.; Kriventseva, E.V.; Zdobnov, E.M. BUSCO: Assessing genome assembly and annotation completeness with single-copy orthologs. Bioinformatics 2015, 31, 3210-3212. [CrossRef] [PubMed]

114. Buchfink, B.; Xie, C.; Huson, D.H. Fast and sensitive protein alignment using DIAMOND. Nat. Methods 2015, 12, 59-60. [CrossRef] [PubMed]

115. Finn, R.D.; Clements, J.; Eddy, S.R. HMMER web server: Interactive sequence similarity searching. Nucleic Acids Res. 2011, 39, W29-W37. [CrossRef] [PubMed]

116. Conesa, A.; Gotz, S.; Garcia-Gomez, J.M.; Terol, J.; Talon, M.; Robles, M. Blast2GO: A universal tool for annotation, visualization and analysis in functional genomics research. Bioinformatics 2005, 21, 3674-3676. [CrossRef] [PubMed]

117. Shimizu, K.; Adachi, J.; Muraoka, Y. ANGLE: A sequencing errors resistant program for predicting protein coding regions in unfinished cDNA. J. Bioinf. Comput. Biol. 2006, 4, 649-664. [CrossRef] [PubMed]

118. Zheng, Y.; Jiao, C.; Sun, H.; Rosli, H.G.; Pombo, M.A.; Zhang, P.; Banf, M.; Dai, X.; Martin, G.B.; Giovannoni, J.J.; et al. iTAK: A program for genome-wide prediction and classification of plant transcription factors, transcriptional regulators, and protein kinases. Mol. Plant 2016, 9, 1667-1670. [CrossRef] [PubMed]

119. Li, A.; Zhang, J.; Zhou, Z. PLEK: A tool for predicting long non-coding RNAs and messenger RNAs based on an improved k-mer scheme. BMC Bioinform. 2014, 15, 311. [CrossRef] [PubMed]

120. Sun, L.; Luo, H.; Bu, D.; Zhao, G.; Yu, K.; Zhang, C.; Liu, Y.; Chen, R.; Zhao, Y. Utilizing sequence intrinsic composition to classify protein-coding and long non-coding transcripts. Nucleic Acids Res. 2013, 41, e166. [CrossRef] [PubMed]

121. Kong, L.; Zhang, Y.; Ye, Z.Q.; Liu, X.Q.; Zhao, S.Q.; Wei, L.; Gao, G. CPC: Assess the protein-coding potential of transcripts using sequence features and support vector machine. Nucleic Acids Res. 2007, 35, W345-W349. [CrossRef]

122. Wu, T.D.; Reeder, J.; Lawrence, M.; Becker, G.; Brauer, M.J. GMAP and GSNAP for genomic sequence alignment: Enhancements to speed, accuracy, and functionality. In Statistical Genomics: Methods and Protocols; Mathé, E., Davis, S., Eds.; Springer: New York, NY, USA, 2016; Volume 1418, pp. 283-334.

123. Alamancos, G.P.; Pages, A.; Trincado, J.L.; Bellora, N.; Eyras, E. Leveraging transcript quantification for fast computation of alternative splicing profiles. RNA 2015, 21, 1521-1531. [CrossRef]

124. Langmead, B.; Salzberg, S.L. Fast gapped-read alignment with Bowtie 2. Nat. Methods 2012, 9, U357-U359. [CrossRef]

125. Dewey, C.N.; Li, B. RSEM: Accurate transcript quantification from RNA-Seq data with or without a reference genome. BMC Bioinform. 2011, 12, 323.

126. Trapnell, C.; Williams, B.A.; Pertea, G.; Mortazavi, A.; Kwan, G.; van Baren, M.J.; Salzberg, S.L.; Wold, B.J.; Pachter, L. Transcript assembly and quantification by RNA-Seq reveals unannotated transcripts and isoform switching during cell differentiation. Nat. Biotechnol. 2010, 28, 511. [CrossRef]

127. Robinson, M.D.; McCarthy, D.J.; Smyth, G.K. edgeR: A Bioconductor package for differential expression analysis of digital gene expression data. Bioinformatics 2010, 26, 139-140. [CrossRef] 
128. Wang, L.; Feng, Z.; Wang, X.; Wang, X.; Zhang, X. DEGseq: An R package for identifying differentially expressed genes from RNA-seq data. Bioinformatics 2010, 26, 136-138. [CrossRef]

129. Storey, J.D. The positive false discovery rate: A Bayesian interpretation and the $q$-value. Ann. Stat. 2003, 31, 2013-2035. [CrossRef]

130. Young, M.D.; Wakefield, M.J.; Smyth, G.K.; Oshlack, A. Gene ontology analysis for RNA-seq: Accounting for selection bias. Genome Biol. 2010, 11, R14. [CrossRef]

131. Mao, X.Z.; Cai, T.; Olyarchuk, J.G.; Wei, L.P. Automated genome annotation and pathway identification using the KEGG Orthology (KO) as a controlled vocabulary. Bioinformatics 2005, 21, 3787-3793. [CrossRef]

132. Tamura, K.; Peterson, D.; Peterson, N.; Stecher, G.; Nei, M.; Kumar, S. MEGA5: Molecular evolutionary genetics analysis using maximum likelihood, evolutionary distance, and maximum parsimony methods. Mol. Biol. Evol. 2011, 28, 2731-2739. [CrossRef]

133. Katoh, K.; Standley, D.M. MAFFT multiple sequence alignment software version 7: Improvements in performance and usability. Mol. Biol. Evol. 2013, 30, 772-780. [CrossRef]

134. Rambaut, A. FigTree v1.4.2: Tree Figure Drawing Tool. 2014. Available online: http://ree.bio.ed.ac.uk/ software/figtree/ (accessed on 6 September 2019).

(C) 2020 by the authors. Licensee MDPI, Basel, Switzerland. This article is an open access article distributed under the terms and conditions of the Creative Commons Attribution (CC BY) license (http://creativecommons.org/licenses/by/4.0/). 